San Jose State University

SJSU ScholarWorks

Master's Theses

Master's Theses and Graduate Research

1991

\title{
Chemically bonded liquid crystals as stationary phases for high performance liquid chromatography
}

Shirish Shah

San Jose State University

Follow this and additional works at: https://scholarworks.sjsu.edu/etd_theses

\section{Recommended Citation}

Shah, Shirish, "Chemically bonded liquid crystals as stationary phases for high performance liquid chromatography" (1991). Master's Theses. 164.

DOI: https://doi.org/10.31979/etd.6mq9-b8gf

https://scholarworks.sjsu.edu/etd_theses/164

This Thesis is brought to you for free and open access by the Master's Theses and Graduate Research at SJSU ScholarWorks. It has been accepted for inclusion in Master's Theses by an authorized administrator of SJSU ScholarWorks. For more information, please contact scholarworks@sjsu.edu. 


\section{INFORMATION TO USERS}

This manuscript has been reproduced from the microfilm master. UMI films the text directly from the original or copy submitted. Thus, some thesis and dissertation copies are in typewriter face, while others may be from any type of computer printer.

The quality of this reproduction is dependent upon the quality of the copy submitted. Broken or indistinct print, colored or poor quality illustrations and photographs, print bleedthrough, substandard margins, and improper alignment can adversely affect reproduction.

In the unlikely event that the author did not send UMI a complete manuscript and there are missing pages, these will be noted. Also, if unauthorized copyright material had to be removed, a note will indicate the deletion.

Oversize materials (e.g., maps, drawings, charts) are reproduced by sectioning the original, beginning at the upper left-hand corner and continuing from left to right in equal sections with small overlaps. Each original is also photographed in one exposure and is included in reduced form at the back of the book.

Photographs included in the original manuscript have been reproduced xerographically in this copy. Higher quality $6 "$ x 9 " black and white photographic prints are available for any photographs or illustrations appearing in this copy for an additional charge. Contact UMI directly to order.

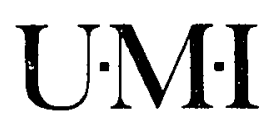

University Microfıms International

A Bell \& Howell Information Company

300 North Zeeb Road. Ann Arbor. MI 48106-1346 USA

$313 / 761-4700 \quad 800 / 521-0600$ 
-.. 
Order Number 1344310

Chemically bonded liquid crystals as stationary phases for high performance liquid chromatography

Shah, Shirish Nagindas, M.S.

San Jose State University, 1991

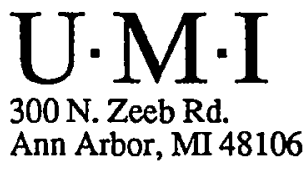




\title{
CHEMICALLY BONDED LIQUID CRYSTALS \\ AS \\ STATIONARY PHASES \\ FOR \\ HIGH PERFORMANCE LIQUID CHROMATOGRAPHY
}

\author{
A Thesis \\ Presented to \\ The Faculty of The Department of Chemistry \\ San Jose State University
}

\author{
In Partial Fulfillment \\ of the Requirement for the Degree \\ Master of Science
}

\author{
by \\ Shirish Shah \\ May, 1991
}


APPROVED FOR THE DEPARTMENT OF CHEMISTRY
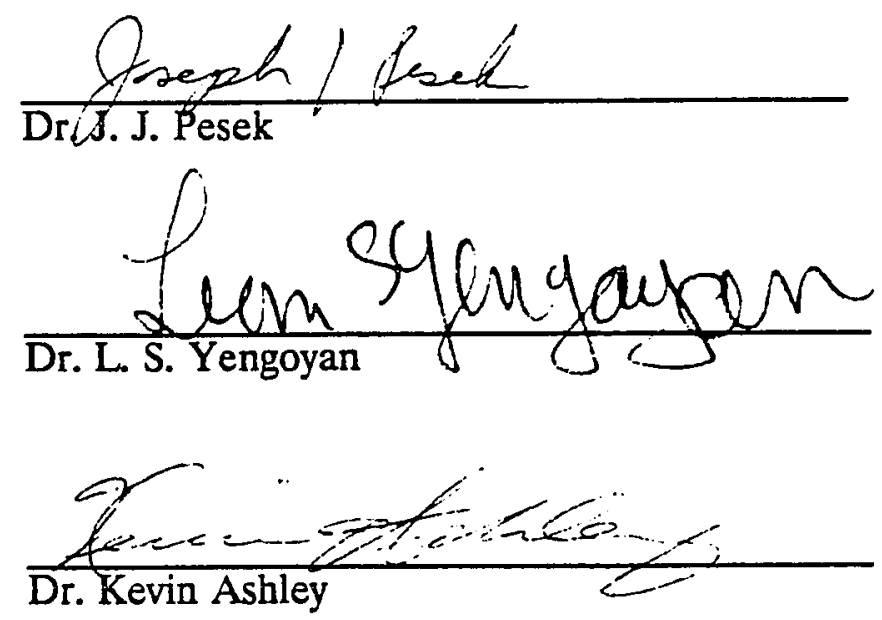

APPROVED FOR THE UNIVERSITY

Serena Mr. Stanford 


\section{ABSTRACT \\ CHEMICALLY BONDED LIQUTD CRYSTALS \\ AS \\ STATIONARY PHASES \\ FOR \\ HIGH PERFORMANCE LIQUID CHROMATOGRAPHY}

by Shirish Shah

A novel synthetic approach used to produce covalently bonded silica-based chromatographic stationary phases is described and discussed. The compound 4-methoxyphenyl 4-allyloxybenzoate (MPAB), known to develop liquid crystalline properties when immobilized onto a polysiloxane backbone, has been bonded to a silica support. Similarly, the liquid crystalline compound cholesteryl 10-undecenoate has also been bonded to such a support. In both the cases, the procedure involves the preparation of a silica intermediate containing stable silicon hydride (silane) surface species, followed by the catalytic addition of these species to the terminal vinyl group present in both esters. Spectroscopic and thermoanalytical evaluation techniques were used to characterize the structure of the modified silica. Diffuse reflectance infrared Fourier transform spectroscopy (DRIFT), differential scanning calorimetry (DSC), electron spectroscopy for chemical analysis (ESCA), and carbon analysis were used to evaluate the modified silica. 


\section{ACKNOWLEDGEMENTS}

I would like to thank Dr. J. J. Pesek for trusting and encouraging me through his patient guidance throughout this work. Also my sincere thanks go to Dr. L. S. Yengoyan and Dr. Kevin Ashley for acting as graduate committee members. I am especially indebted to Dr. J. E. Sandoval for his critical reading of the entire manuscript and for many improvements he suggested. Finally a special note of thanks to Dr. Biringer for his time and valuable suggestions.

Shirish Shah

$03 / 25 / 91$ 


\section{TABLE OF CONTENTS}

\section{INTRODUCTION}
A. Background $\ldots \ldots \ldots \ldots \ldots \ldots \ldots \ldots \ldots \ldots \ldots \ldots \ldots \ldots \ldots \ldots$
B. Development of bonded stationary phases $\ldots \ldots \ldots \ldots \ldots \ldots .2$
C. Liquid crystais $\ldots \ldots \ldots \ldots \ldots \ldots \ldots \ldots \ldots \ldots \ldots \ldots \ldots \ldots \ldots$
D. Liquid crystais and chromatography $\ldots \ldots \ldots \ldots \ldots \ldots \ldots \ldots \ldots \ldots$

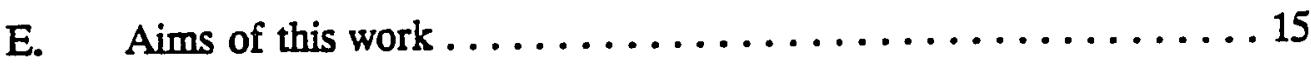

\section{EXPERIMENTAL}

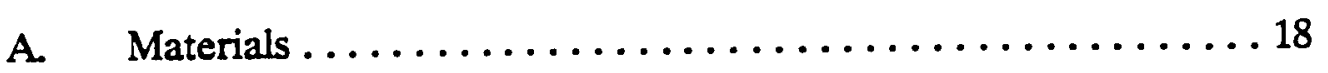

B. Apparatus and equipments $\ldots \ldots \ldots \ldots \ldots \ldots \ldots \ldots \ldots \ldots \ldots \ldots$

C. Synthesis of 4-methoxyphenyl 4-allyloxybenzoate (MPAB) $\ldots \ldots \ldots 20$

1. Preparation of 4-(allyloxy) benzoic acid

2. Preparation of 4-(allyloxy) benzoyl chloride

3. Preparation of 4-methoxyphenyi 4-allyloxybenzoate (MPAB)

D. Synthesis of dicyciopentadienyl platinum (II) chloride ........23

E. Preparation of hydrided silica $\ldots \ldots \ldots \ldots \ldots \ldots \ldots \ldots \ldots$

1. Chlorination/reduction sequence

a. Chlorination of silica

b. Reduction of chlorinated silica

2. Silanetriol condensation on silica

F. Hydrosilation of MPAB on hydrided silica $\ldots \ldots \ldots \ldots \ldots 25$

G. Hydrosilation of cholesteryl 10-undecenoate on hydrided silica . . . 26 


\section{RESULTS AND DISCUSSION}

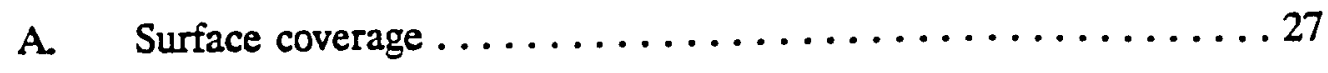

B. Effect of catalyst on hydrosilation $\ldots \ldots \ldots \ldots \ldots \ldots \ldots \ldots \ldots \ldots \ldots \ldots$

C. Effect of time on surface coverage $\ldots \ldots \ldots \ldots \ldots \ldots \ldots \ldots \ldots \ldots$

D. Effect of hydride intermediate on surface coverage $\ldots \ldots \ldots \ldots 33$

E. Spectroscopic evaluation $\ldots \ldots \ldots \ldots \ldots \ldots \ldots \ldots \ldots \ldots \ldots \ldots \ldots \ldots \ldots \ldots$

1. Native silica

2. Hydride intermediate

3. 4-Methoxyphenyl 4-allyloxybenzoate (MPAB)

4. Cholesteryl 10-undecenoate

F. Thermal characterization ......................49

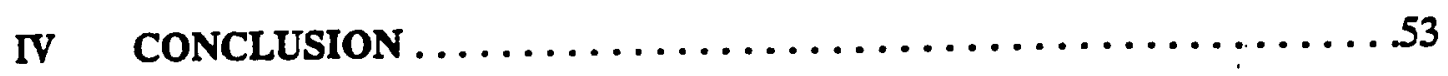

REFERENCES 


\section{LIST OF FIGURES}

Figures

Page

1) Idealized representation of liquid crystals: a) Smectic $A$, b) Smectic $C$, c) Nematic, and d) Cholesteric.

2) Silanization of liquid crystal on silica

3) Reaction scheme for the synthesis of liquid crystalline polymeric stationary phases.

4) Structural formula of liquid crystals used in this work.

5) Schematic diagram of preparation of MPAB.

6) Effect of catalyst on surface coverage. platinum (II) chloride and hexachloroplatinic acid.

7) Partial DRIFT spectral comparison of cholesteryl 10-undecenoate bonded to silica: $A$ ) bonded phase prepared by using hexachloroplatinic acid, and $B$ ) by using dicyclopentadienyl platinum (II) chloride

8) Surface coverage as a function of time.

9) Effect of intermediate on surface coverage.

ए203 Chlorination/reduction product and silanetriol condensation product.

10) DRIFT spectrum of native silica.

11) DRIFT spectrum of hydrided silica.

12) DRIFT spectrum of 4-methoxyphenyl 4-allylaxybenzoate.

13) DRIFT spectrum of cholesteryl 10-undecenoate.

14) Partial DRIFT spectra of native silicas and hydrided intermediates: $A$ and $C$ are the specra of native silicas from two different batches. $B$ and $D$ are hydrided intermediates, respectively. 
15) Partial DRIFT spectrum of silane intermediate prepared by chlorination/reduction technique $(A)$, and $(B)$ after bonding with 4-methoxyphenyl 4-allyloxybenzoate.

16) Partial DRIFT spectnum of silane intermediate prepared by silanetriol condensation technique $(A)$, and $(B)$ after bonding with 4-methoxyphenyl 4-allyloxybenzoate.

17) Partial DRIFT spectra of hydrided silica bonded with 4-methoxyphenyl 4-allyloxybenzoate: $(A)$ in hydride intermediate prepared via chlorination/reduction technique, and $(B)$ in hydride intermediate prepared by silanetriol condensation process.

18) Partial DRIFT spectrum of silane intermadiate prepared by chlorination/reduction technique $(A)$, and $(B)$ after bonding with cholesteryl 10-undecenoate.

19) Partial DRIFT spectrum of silane intermediate prepared by silanetriol condensation technique $(A)$, and $(B)$ after bonding with cholesteryl 10-undecenoate.

20) Partial DRIFT spectra of hydrided silica bonded with cholesteryl 10-undecenoate: $(A)$ in hydride intermediate prepared via chlorination/reduction technique, and $(B)$ in hydride intermediate prepared by silanetriol condensation process.

21) DSC thermogram of 4-methoxyphenyl 4-allyloxybenzoate.

22) . DSC thermogram of cholesteryl 10-undecenoate.

23) DSC thermogram of 4-methoxyphenyl 4-allyloxybenzoate bonded silica. 


\section{LIST OF TABLES}

Table

Page

1) Hydrosilation of liquid crystals on hydrided silica using two different catalytic systems.

2) Surface coverage as a function of reaction time. Cholesteryl 10-undecenoate on hydrided silica.

3) Surface coverage as a function of time. 4-Methaxyphenyl

32 4-allyloxybenzoate on hydrided silica.

4) Hydrided vs silane deposited intermediate. 


\section{CHAPTER I}

\section{INTRODUCTION}

\section{A. Background}

Separation methods are an important analytical tool and chromatography has developed into the premier separation technique. Chromatography is a method of separation belonging to the class of diffusion mass transfer operations where the components of a mixture are separated as a result of their partitioning between two immiscible heterogeneous phases. Prior to the development of chromatographic techniques, distillation was the only technique available for the separation of two or more miscible components. Chromatographic methods were first used for analytical purposes to study certain complex natural and synthetic substances that were incapable of examination by other methods. For instance, a chromatographic method was used to clarify sugar solutions as far back as the middle of last century.

The new technique, gas chromatography (GC) was found to be simple, fast, and capable of producing separations of volatile materiais that were impossible by distillation. Additionally, automated GC was soon available for convenient, fast, and unattended operation. However, despite its tremendous capabilities, GC is unable to directly handle samples which are non-volatile and/or thermally labile. Liquid chromatography (LC), on the other hand, is very well suited for the 
separation of macromolecules and ionic solutes, not only because of its lower separation temperature, but also because of its superior selectivity (two phases for interactions in LC vs only one in GC) and availabllity of a great variety of stationary phases. The use of the acronym HPLC (high performance liquid chromatography) became more frequent where "high performance" refers to a high speed and high efficiency separation. Although LC had its origin in the late 50's, it was not until the late 60 's when its potential started to be widely recognized. Since then, tremendous activity has been aimed at the development of separation theory and equipment.

\section{B. Development of bonded stationary phases}

Silica and chemically modified versions of silica have been used widely as supports in separation science, especially in chromatography. Silica can be considered as the polymerization product of silicic acid. Silica is a weak acid having a pKa value of about 7, and is sparingly soluble in water at pH's ranging between 2 and 8; but above pH 8 its solubility increases dramatically due to depolymerization. Silica is constructed from a three dimensional network of siloxane moieties (-Si-O-Si-) with silanol groups (-Si-OH) at the surface. Porous silica has long been used as an adsorbent or as a support for stationary phases in chromatography.

With the advancement of the field of chromatography, preparation of 
stationary phases by attaching surface groups has received considerable attention. An esterification reaction was carried out between silanols and an alcohol to form Si-O-C surface linkages $[1,2]$

$=\mathrm{Si}-\mathrm{OH}+\mathrm{R}-\mathrm{OH} \longrightarrow \mathrm{Si}-\mathrm{O}-\mathrm{R}+\mathrm{H}_{2} \mathrm{O}$

Due to their poor hydrolytic stability, these ester-type bonded phases currently have little, if any, use. A widely accepted means of preparing bonded phases has been the attachment of organic moieties to silica surfaces via organosilane molecules. Alkylchlorosilanes are among the most commonly used reagents for silanization [3]. The alkylchlorosilane reacts with the hydroxyl functional group to form a wide variety of bonded phases. A typical reaction scheme can be represented as<smiles>[R][Si](C)(Cl)O[Si][Si]([R])(C)C</smiles>

where $\mathrm{R}$ ranges from non-polar groups such as $\left(\mathrm{CH}_{2}\right)_{n} \mathrm{CH}_{3}$ with $\mathrm{n}=7$ to 17 (used in reversed phase liquid chromatography) to ionic groups such as benzenesulfonic acid (used in ion-exchange liquid chromatography). 
The reproducibility and extent of bonding reaction usually require a close control of various parameters like reaction time, reaction temperature, concentration of reactants and inertness of reaction atmosphere. This has been true especially for reversed phase materials. A still limited hydrolytic stability has always been a bottleneck in liquid chromatography, especially in cases when the mobile phase contains a low or high $\mathrm{pH}$ aqueous component. This limitation has resulted in the development of new synthetic routes, such as those involving the formation of direct Si-C bonds, which are thought to provide more stable bonded phases.

There are several methods available for direct silicon-carbon linkages. The Si-C bonds are prepared by the reaction between a Grignard or organolithium reagent and a silicon halide. A chlorination/alkylation reaction scheme has been reported to synthesize non-polar bonded stationary phases for gas chromatography $[2,4,5,6]$. A typical reaction scheme can be represented as

$$
\begin{aligned}
& \equiv \mathrm{Si}-\mathrm{OH}+\mathrm{SOCl}_{2} \rightarrow \equiv \mathrm{Si}-\mathrm{Cl}+\mathrm{SO}_{2}+\mathrm{HCl} \\
& \equiv \mathrm{Si}-\mathrm{Cl}+\mathrm{RMgBr} \rightarrow \mathrm{Si}-\mathrm{R}+\mathrm{MgBrCl} \\
& \text { or } \\
& \equiv \mathrm{Si}-\mathrm{Cl}+\mathrm{Li}-\mathrm{R} \longrightarrow \cong \mathrm{Si}-\mathrm{R}+\mathrm{LiCl}
\end{aligned}
$$

Widespread acceptance of the chlorination/Grignard or chlorination/organolithium route has been difficult because of the residual salts 
trapped in the silica matrix and very stringent reaction conditions. Perhaps more importantly, alkylation reagents are very reactive towards carbonyl, nitrile, carboxyl, amide, and alcohol groups, therefore limiting the applicability of this particular reaction scheme. One of the prime requirements here is that the organic group $R$ must remain inert and intact during the preparation of the reagents. It is an established fact that acidic components react with Grignard reagents to form the corresponding hydrocarbon R-H. "Any compound containing hydrogen attached to an electronegative element such as oxygen, nitrogen, and even triply bonded carbon are acidic enough to decompose a Grignard agent" [7]. These alkylation reactions are very air sensitive as they react instantaneously with molecular oxygen, carbon dioxide, and with nearly every organic compound containing a C-O or C-N multiple bond. The above restrictions put a limit to the number of functionalities in $R$ that can be used to prepare a Grignard reagent.

Recently, an alternate route has been developed to produce a stable Si-C linkage at the silica surface [8]. It involves the preparation of a stable intermediate containing $\mathrm{Si}-\mathrm{H}$ species, followed by the catalytic addition to the $\mathrm{Si}-\mathrm{H}$ species of the terminal vinyl group of an organic moiety. One way of preparing the intermediate involves the chlorination at the silica surface followed by the reduction of the resulting $\mathrm{Si}-\mathrm{Cl}$ group to $\mathrm{Si}-\mathrm{H}$ species.

$$
\exists \mathrm{Si}-\mathrm{OH}+\mathrm{SOCl}_{2} \longrightarrow \mathrm{ISi}-\mathrm{Cl}+\mathrm{SO}_{2}+\mathrm{HCl}
$$


$4 \equiv \mathrm{Si}-\mathrm{Cl}+\mathrm{LiAlH}_{4} \longrightarrow 4 \equiv \mathrm{Si}-\mathrm{H}+\mathrm{LiCl}+\mathrm{AlCl}$

Alternatively, a milder reducing agent such as $\operatorname{Red} \mathrm{Al}^{\bullet}$ (Solution of Bis(2methoxyethoxy) aluminum hydride) can be used.

$2 \equiv \mathrm{Si}-\mathrm{Cl}+\left[\left(\mathrm{CH}_{3} \mathrm{OCH}_{2} \mathrm{CH}_{2} \mathrm{O}_{2} \mathrm{AlH}_{2}\right] \mathrm{Na} \longrightarrow 2 \equiv \mathrm{Si}-\mathrm{H}\right.$

Current development for the production of the silica intermediate has resulted in the use of a silane reagent in a fashion similar to that employed in the so-called "silane coupling chemistry" $[9,10]$.

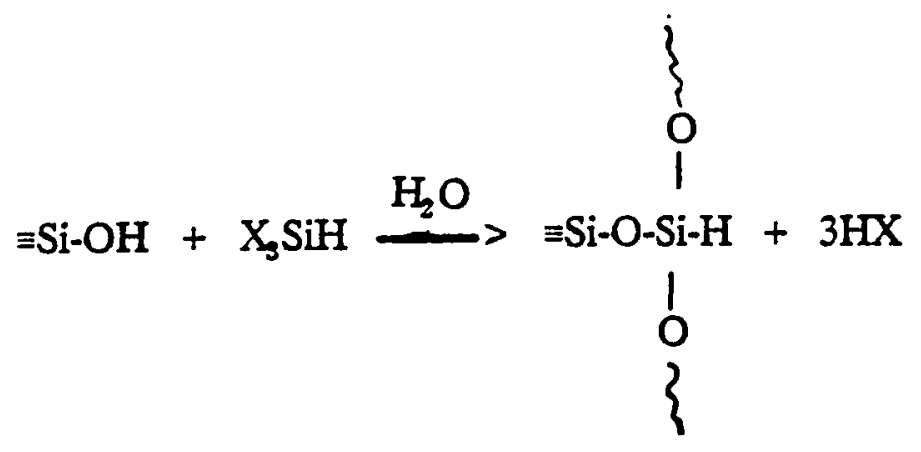

The group X can be easily hydrolyzed and typically consist of an acyloxy, amine, chlorine, or alkoxy group. The common alkoxy groups like methoxy and ethoxy give methanol and ethanol as byproducts, respectively. The reaction consists of several steps. Hydrolysis of the silane occurs upon water addition; this is followed by the formation of oligomers via a condensation reaction. The oligomers form hydrogen bonds with the OH groups of the substrate's silanols. Further silanol condensation will result in the formation of a covalent linkage between the silane and 
the surface.

The application of silicon hydride addition to olefins and acetylenes, discovered in the late 1940's, has proved to be a feasible way of synthesizing new stationary phases with silicon-carbon bonding. The reaction in general can be represented as

$$
\equiv \mathrm{Si}-\mathrm{H}+\mathrm{CH}_{2}=\mathrm{CHR} \longrightarrow \quad \equiv \mathrm{Si}-\mathrm{CH}_{2}-\mathrm{CH}_{2}-\mathrm{R}
$$

This seems to be a very effective route for attaching organic moeities containing active functional groups to silicon atoms. This process is called hydrosilation. It can be achieved either thermally, simply by heating the reactants together under pressure, or in the presence of a catalyst.

\section{Liquid crystals}

The term "liquid crystals" first used by O. Lehmann in 1890, designates a state of matter that is intermediate between solid crystalline and the ordinary (isotropic) liquid phase. The existence of a liquid crystalline state was first observed by Reinitzer while synthesizing cholesteryl benzoate [11]. He found that, on heating the compound, it melted at $150^{\circ} \mathrm{C}$ as a turbid liquid which became a clear, isotropic liquid at $179^{\circ} \mathrm{C}$. Liquid crystals flow like ordinary fluids; i.e., they adopt the shape 
of the container. On the other hand, they exhibit anisotropic properties, as do solid crystals. Liquid crystals can be defined as "condensed fluid phases with spontaneous anisotropy" [11]. Liquid crystals are also called mesophases or mesomorphic phases because of their intermediate nature. A mesomorphic phase can thus be defined as a thermodynamically stable anisotropic liquid.

Over the past 25 years liquid crystals have attracted much interest and considerable progress has been made with respect to knowledge in this field. Due to their unusual properties, liquid crystals are used in many different disciplines of science and engineering. A very unusual property - the color change that they can undergo - has found widespread application. Once mere chemical curiosities, they are now common as an essential material in many electronically controlled displays. The numerical displays on watches, pocket calculators, thermometers, games, and many other electronic and electrical devices depend on them. Liquid crystals also found application in forming television pictures, displays for telephone, automobile dashboards, etc. They appear to be ideal as displays for portable instruments incorporating microprocessors and for windows and panels in the construction industry.

Liquid crystals can be classified as thermotropic and lyotropic. When a thermotropic crystal is heated the liquid crystalline state appears upon melting and the liquid retains this property over a certain temperature range. Upon further 
heating one or more transitions may take place until a certain temperature, called the "clearing" temperature, is reached. The temperature difference between the melting point and the clearing temperature indicate the mesophase range of that particular liquid crystal. Lyotropic liquid crystals exhibit liquid crystalline properties in a suitable solvent or solvent mixture over a wide range of concentration. They can be obtained by mixing two or more components. Usually they are surface active agents or polymers. These compounds are amphiphilics, i.e. compounds having two different regions, one hydrophobic and the other hydrophilic (e.g., soaps, detergents and certain polypeptides) [12]. Compounds that exhibit thermotropic liquid crystalline behavior are non-amphiphilic. Most of the research work in gas chromatography has been done with thermotropic liquid crystals.

In general, non-amphiphilic liquid crystalline materials are elongated, rodlike, fairly rigid structures which usuaily can be represented as

\section{$Y-A R-X-A R$ ' $Y$}

where $A R$ and $A R$ ' are aromatic structures with one or more rings that are polarizable, planar, and rigid, $\mathrm{X}$ is the central atom capable of conjugating electronically with the aromatic nuclei and $Y$ and $Y^{\prime}$ are para-terminal substituents. $\mathrm{X}$ - ray spectroscopic studies have proved that the rod-like molecules tend to pack as in solid crystal lattice with their long axes parallel. The liquid crystals that are 
used as stationary phases contain a variety of organic functionalities such as Schiff bases, esters, isocyanates, azo and azoxy compounds, biphenyis and terphenyl derivatives.

Depending on the molecular orientation, liquid crystals can be classified as nematic, cholesteric, and smectic type (Figure 1).

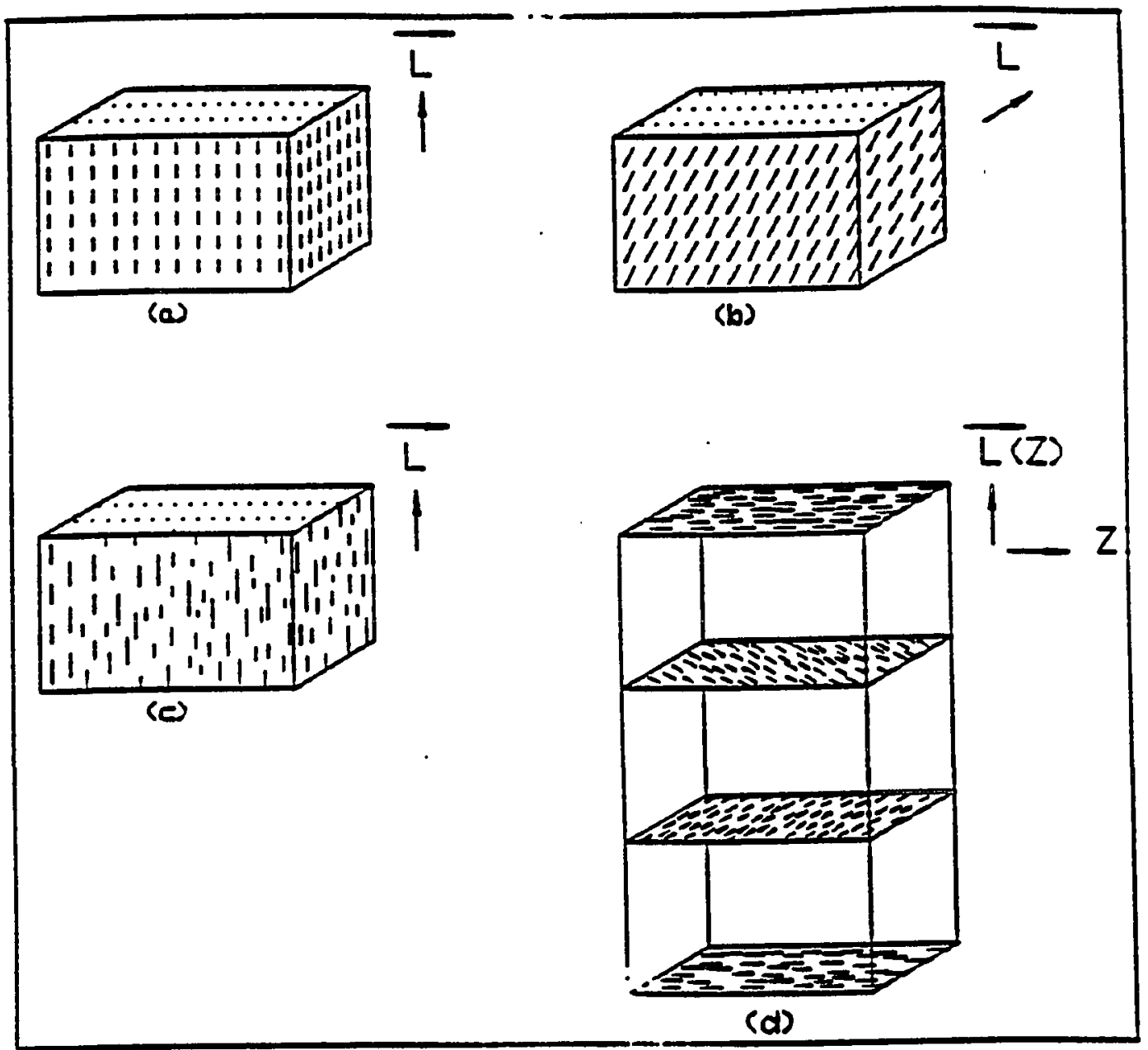

Figure 1. Idealized representation of liquid cystals: a) Smectic A, b) Smectic $C$, c) Nematic, and d) Cholesteric. 
Nematic liquid crystals are characterized by a long molecular axis which is aligned parallel to the preferred direction $\bar{I}$. The centers of gravity are distributed at random as in an ordinary liquid. This type of mesomorphic behavior is found in compounds with elongated, rod-like molecules consisting of a conjugated aromatic system. Nematic liquid crystals have found very useful applications in electro-optical devices.

Cholesteric liquid crystals are best described as a twisted nematic structure. If we consider a certain plane, the molecules are aligned parallel to the preferred direction $\overline{\mathrm{L}}$ as in nematic phase while proceeding in a direction normal to the plane, L rotates continuously, resulting into a helical structure. Since cholesteric liquid crystals usually contain a center of optical activity, the rotation could be left-handed (type "laevo") or right handed (type "dextro"). Very often, cholesteric materials are derivatives of cholesterol. However, it should be pointed out that neither is this type of liquid crystal restricted to members in the cholesteryl group, nor do all cholesteryl derivatives show a cholesteric phase. It had been just a fortuitous case that for many years only cholesteryl derivatives exhibited cholesteric behavior. Cholesteric esters usually exhibit liquid crystalline properties in the range of $20^{\circ} \mathrm{C}-100^{\circ} \mathrm{C}$.

Smectic liquid crystals have a layered structure. The centers of gravity of the elongated molecules are arranged in equidistant planes. The long axes of the molecules are parallel to a preferred direction $\overline{\mathrm{L}}$ which may be normal to the plane 
(Smectic $\mathrm{A}$ ) or tilted by a certain angle (Smectic $\mathrm{C}$ ). The arrangement of the center of the gravity within the plane may be random or regular. Molecular ordering of a liquid crystal could be determined by $\mathrm{X}$ - ray analysis.

A great deal of information is available about the morphological behavior of smectic, nematic, and cholesteric phases in the range of their transition point(s). Numerous properties of liquid crystals such as density, viscosity, surface tension, vapor pressure, and coefficient of thermal expansion are known to undergo significant changes in this region [13].

\section{Liquid crystals and chromatography}

Within the sphere of analytical chemistry, use of liquid crystals as stationary phases in gas chromatography (GC) has been well known for over 25 years. Some of them possess unique properties with respect to the separation of complex organic molecules. Liquid crystals should have a high thermal stability in order to be used as a stationary phase in GC. They should not decompose at normal column operating temperature and should possess a low vapor pressure so that the quantity of the phase in the column is not reduced to a degree that affects the reproducibility of the results. Another desirable property is a wide mesophase temperature range in order to facilitate the programming of the column. 
The unique separating capabilities of liquid crystals are due to the structure and ordering of their molecules. In their mesomorphic temperature range - from melting point to the point of transition to the isotropic liquid phase - liquid crystals have orientational molecular ordering. It is a well known fact now that liquid crystalline bonded phases have an added dimension of selectivity for chromatographic separations [12]. Interactions resulting from the ordered compact packing of the liquid crystalline material and the solute add a unique feature to the separation mechanisms. The separation of geometrical isomers results in an elution order that approximately follows length to breadth ratio and the planarity of the solute. The use of a liquid crystal as a stationary phase was first reported by Kelkar $[14,15]$ and simultaneously by Dewar and Schroeder $[16,17]$. They can be employed as a sorbent in gas chromatography for the separation of organic isomers $[18,19]$, polynuclear hydrocarbons (PAH) [20], and optical isomers [21]. Liquid crystals are physically coated on a solid substrate or on the wall of a capillary tube in gas chromatographic applications. The low volatility of liquid crystals results in a long life span.

In HPLC, prolonged exposure to the mobile phase and shear forces due to high pressure could result in rapid loss of a physically coated liquid crystalline material which in turn result in a decrease of separation efficiency. There are not many references available for their application in HPLC [22,23]. The only practical solution in HPLC is to produce chemically bonded materials that possess liquid crystal properties. Attempts made to bond cholesteric ester liquid crystals for use in 
HPLC as a stationary phase have been unsuccessful [24]. Liquid crystals bonded to polysiloxane polymers have been shown to retain their mesophase characteristics [25]. The material 4-methoxyphenyl-4-allyloxybenzoate (MPAB) was shown to retain liquid crystalline properties when bonded to a polysiloxane backbone [26]. Similar behavior can be expected of liquid crystal materials covalently bonded to silica. A liquid crystalline bonded silica was recently prepared [27]. The reaction scheme can be summarized as follows
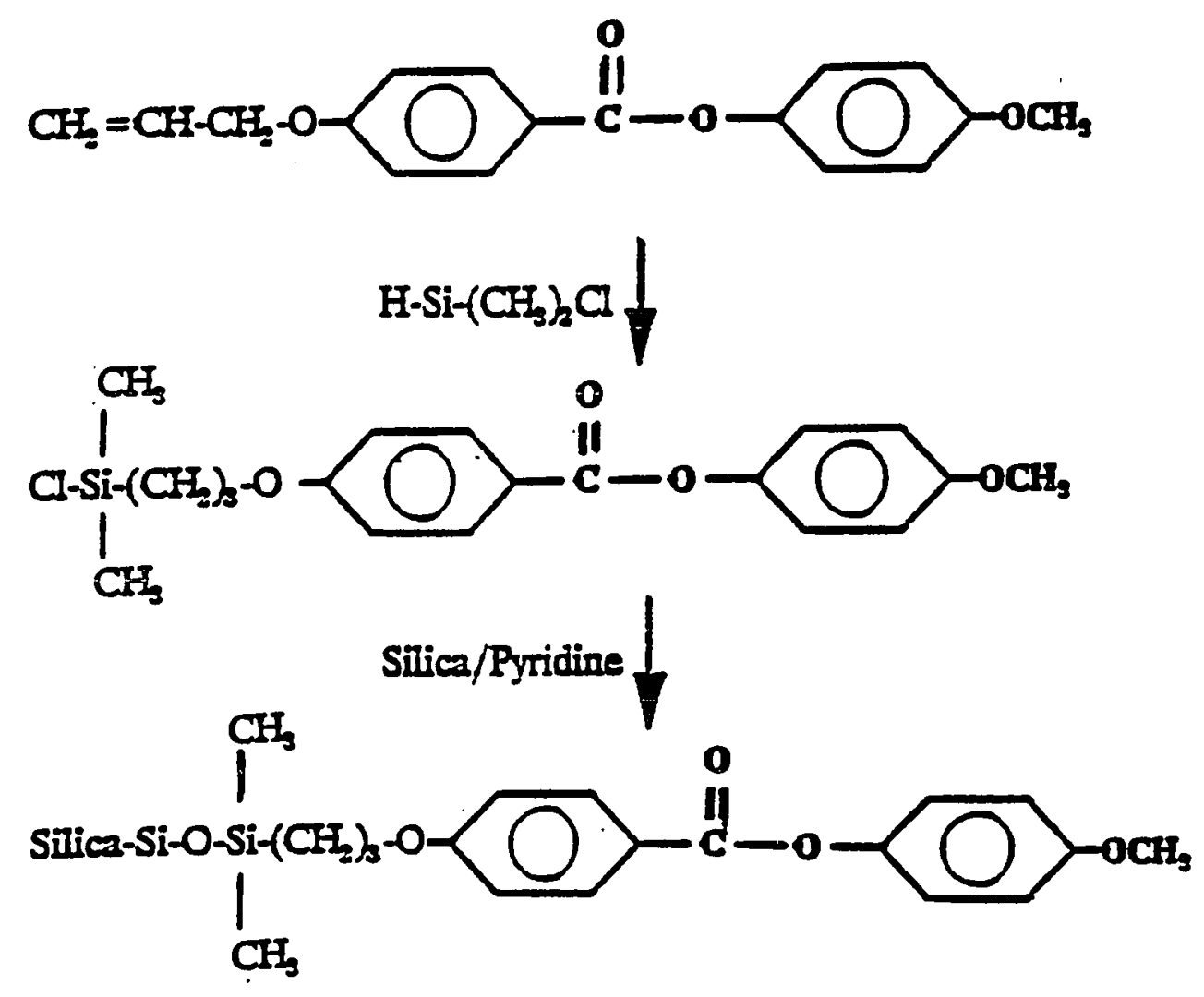

Figure 2. Silanization of liquid crystal on silica 
E. Aims of this work

Figure 3 shows the reaction scheme followed by Laub and co-workers [26] for the synthesis of liquid crystalline polymeric stationary phases.

$\left(\mathrm{H}_{3} \mathrm{C}_{3}-\mathrm{Si}-\mathrm{O}\left[\begin{array}{c}\mathrm{CH}_{3} \\ \mathrm{Si}-\mathrm{O} \\ 1 \\ \mathrm{H}\end{array}\right]_{\mathrm{n}} \mathrm{Si}-\left(\mathrm{CH}_{3}\right)_{3}+\mathrm{CH}=\mathrm{CH}-\left(\mathrm{CH}_{2}\right)_{2}-\mathrm{R}\right.$

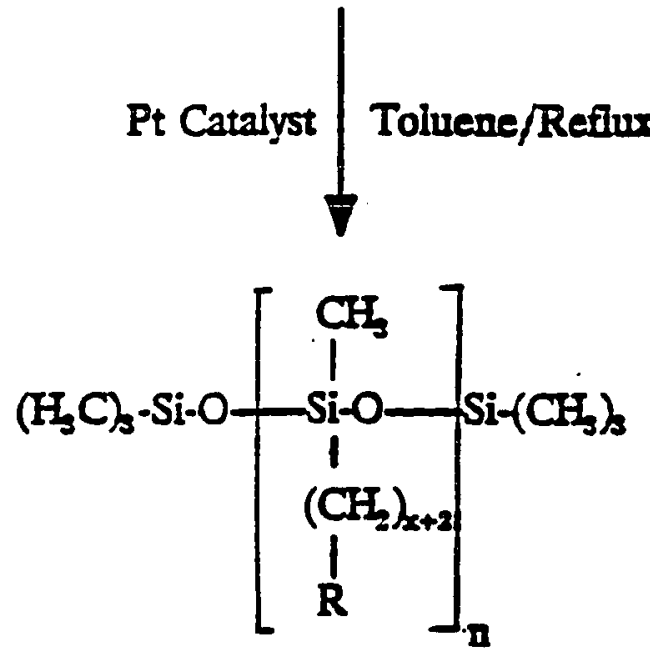

Figure 3. Reaction scheme for the synthesis of liquid crystalline polymeric stationary phases.

Excellent separations of PAH's with these phases were readily accomplished. The most important feature of these products is perhaps the fact that the liquid crystalline esters used did not lose their mesomorphic properties when immobilized on the polymeric backbone. Moreover, in one instance a regular (isotropic) compound was found to develop liquid crystalline behavior upon attachment to a 
polymeric chain. The potential for attachment of vinyl terminated liquid crystalline compounds to a silica support which contains silica hydride surface species is thus evident. The same fundamental approach this time on a hydrided silica surface is used in this work with two different kinds of compounds. One phenyl ester and one cholesteryl ester are used in this investigation. Both compounds selected are vinyl terminated with a general formula $\mathrm{CH}_{2}=\mathrm{CH}-\mathrm{R}$ providing an active bonding site. The structural formula of both compounds are given below.

1) 4-Methoxyphenyl 4-allyloxy benezoate

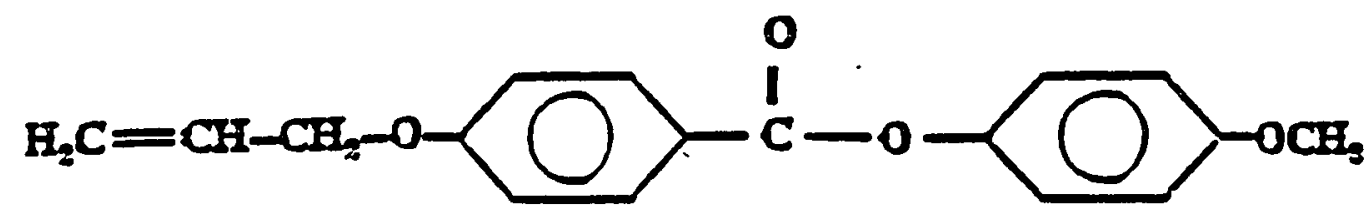

2) Cholesteryi 10-undecenoate

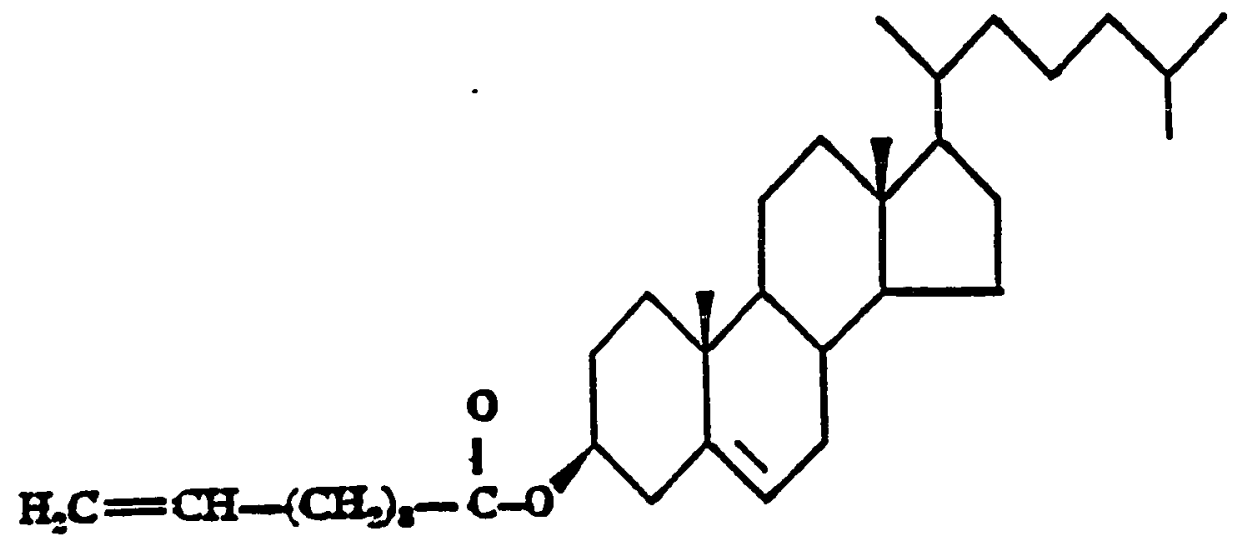

Figure 4. Structural formula of liquid crystals used in this work 
The research contained in this thesis describes the preparation of liquid crystals and its chemical bonding to a porous silica substrate containing surface hydride groups. This hydride intermediate is produced by either the chlorination/ reduction reaction sequence or through the surface deposition of silanetriol groups. Liquid crystals with a terminal olefinic group are used to provide active sites for surface addition, which should result in a direct linkage of the liquid crystals to the silica surface. The surface binding can be schematically represented as

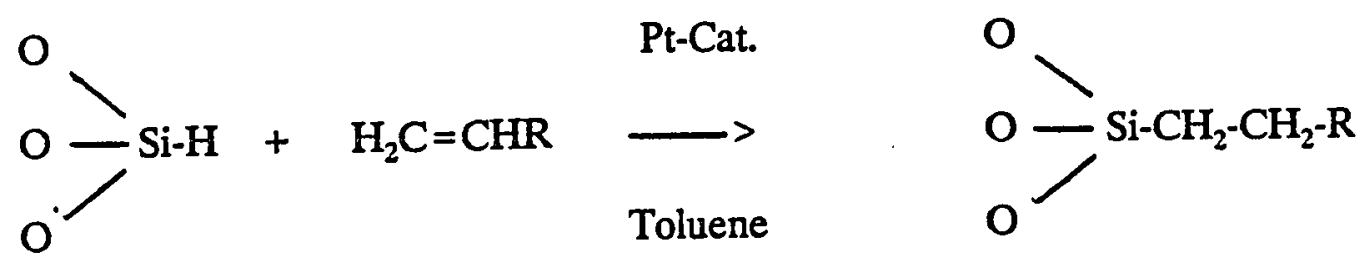

Chemically bonded liquid crystal stationary phases are analyzed using various analytical techniques like diffuse reflectance infrared Fourier transform (DRIFT) spectrophotometry, electron spectroscopy for chemical analysis (ESCA), and differential scanning calorimetry (DSC). 


\section{CHAPTER II}

\section{EXPERIMENTAL}

\section{A. Materials}

4-Hydroxybenzoic acid, allyl bromide (3-bromopropene), 4-methoxyphenol (p-methoxyphenol) and thionyl chloride were reagent grade; they were used without further purification. 4-Methoxyphenyl 4-allyloxybenzoate was prepared as described by Laub and co-workers [26]. Cholesteryl 10-undecenoate was purchased from Chemical Dynamics Corporation and Schweizerhall Inc., (N.J.). Partisil ${ }^{\bullet}$ silica with particle size of $40 \mathrm{\mu m}$, average pore diameter of $90 \AA$ and specific surface area of $315.3 \mathrm{~m}^{2} / \mathrm{g}$ was obtained from Alltech Associates, Deerfield, IL. and was dried at $120^{\circ} \mathrm{C}$ in a vacuum oven overnight before using. Nucleosil ${ }^{\bullet} 300-10$ silica, purchased from Alltech Associates, Deerfield, IL. with a particle size of $10 \mu \mathrm{m}$, average pore diameter of $393 \AA$ and $83.2 \mathrm{~m}^{2} / g$ specific surface area was dried in the same manner before use. Toluene, tetrahydrofuran (THF), and dioxane solvents were dried by refluxing over calcium hydride for several days and freshly distilled as needed. Pyridine was dried by refluxing over $\mathrm{KOH}$ pellets for 5 days under a nitrogen stream. A 3.4M Red-Al ${ }^{\circ}$ (solution of sodium bis(2-methoxyethoxy) aluminum hydride in toluene) was diluted 10 -fold and used as the reducing agent. Dicyclopentadienyl platinum (II) chloride was prepared as described by Laub and co-workers [26]. Triethoxysilane and chloroplatinic acid was purchased from Aldrich Chemical Co., 
Milwaukee, WI. and used as received. Deionized water was obtained from a Milli-Q ${ }^{\infty}$ (Millipore Corporation, Milford, MA) water system.

\section{B. Apparatus and equipments}

Differential scanning calorimetry (DSC) and thermal gravimetric analysis (TGA) were performed with a Du Pont 2100 Thermal analysis system. Fourier transform infrared spectra were taken with a model 1800 (Perkin Elmer, Norwalk, CT) spectrometer equipped with a deuterated triglycine sulfate (DTGS) detector. A diffuse reflectance accessory (Spectra Tech., Stamford, CT) with a 2-mm diameter, 2-mm depth sample cup was used. The samples were prepared by diluting the silica with finely ground $\mathrm{KBr}$ powder $(1: 1 \mathrm{wt} / \mathrm{wt})$. Spectra were collected at $2 \mathrm{~cm}^{-1}$ resolution after 100 sample scans ratioed against pure $\mathrm{KBr}$ as reference. Carbon analysis was done at the Chemistry Department of University of California, Berkeley, CA. Electron spectroscopy for chemical analysis (ESCA) was done at Surface Science Lab., Mountain View, CA. Specific surface area $\left(\mathrm{m}^{2} / \mathrm{g}\right)$ determinations were performed at the Chevron Research Center Richmond, CA using the Brunauer, Emmet, and Teller (BET) nitrogen adsorption method. 
C. Synthesis of 4-methoxyphenyl 4-allyloxybenzoate (MPAB)

4-Methoxyphenyl 4-allyloxybenzoate was prepared according to the procedure developed by Laub and co-workers [26]. Figure 5 summarizes the various steps involved.

\section{STEP 1}

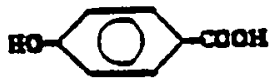

a
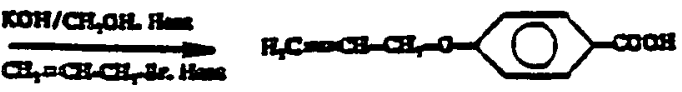

\section{STEP 2}

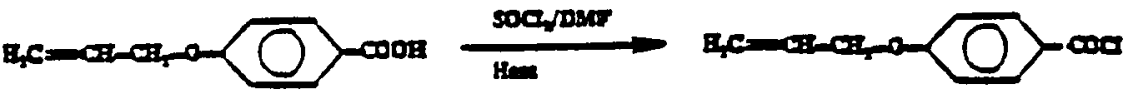

\section{STEP 3}
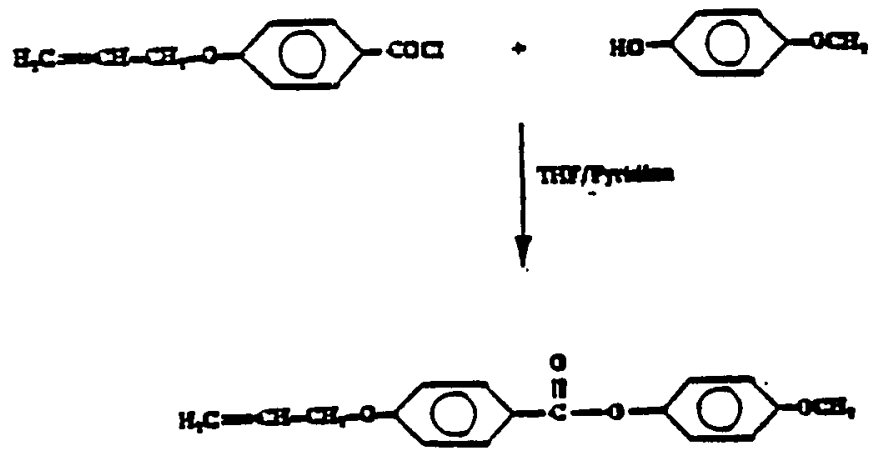

Figure 5. Schematic diagram of preparation of $M P A B$. 


\section{Preparation of 4-(allyloxy)benzoic acid}

A 3-neck, 1-liter round bottom flask equipped with an addition funnel, a heating mantle and magnetic stir bar was charged with $90.00 \mathrm{~g}$ ( 0.65 moles) of 4-hydroxybenzoic acid, followed by the addition of $400 \mathrm{~mL}$ of methanol at room temperature. A clear yellowish solution was obtained. In a separate flask containing $125 \mathrm{~mL}$ of deionized water, $105.00 \mathrm{~g}$ (1.9 mole) of $\mathrm{KOH}$ were added. This $\mathrm{KOH}$ solution was added dropwise to the main reaction flask. Due to the exothermic nature of the reaction a reflux was observed. Heating started to maintain a gentle reflux and $90.00 \mathrm{~g}$ ( 0.74 moles) of allyl bromide was added dropwise over a 1 hour period. The reaction was allowed to proceed under reflux for 8 hours and then about $300 \mathrm{~mL}$ of methanol were evaporated off to reduce the volume. $100 \mathrm{~mL}$ of water was added and the reaction mixture cooled to room temperature. The aqueous layer was extracted with three $150 \mathrm{~mL}$ portions of diethyl ether and the remaining aqueous layer was neutralized with $20 \%$ (v/v) $\mathrm{HCl}$. The resulting creamish precipitate was filtered, dried and weighed (103.42 g). After recrystallizing from ethanol the final product exhibited a m.p. of $164^{\circ} \mathrm{C}-166^{\circ} \mathrm{C}$. Final yield was $80.4 \mathrm{~g}(70 \%)$.

\section{Preparation of 4-(allyloxy)benzoyl chloride}

$25.00 \mathrm{~g}$ (0.14 moles) of 4-(allyloxy)benzoic acid prepared as above was placed in a 100-mL round bottom flask, equipped with a reflux condenser, a drying tube, magnetic stirrer and a heating bath. $25 \mathrm{~mL}$ ( 0.21 moles) of thionyl chloride $\left(\mathrm{SOCl}_{2}\right)$ 
and 10 drops of dimethyl formamide (DMF) were added to the flask. The reaction mixture was stirred overnight at room temperature under a nitrogen atmosphere. It was then heated to $60^{\circ} \mathrm{C}$ for two hours to ensure the completion of the reaction. The excess of thionyl chloride was distilled off and the crude product was distilled in vacuum at $100^{\circ} \mathrm{C} / 1$ torr. yielding $19.00 \mathrm{~g}(70 \%)$ of a pale yellow liquid as final product.

3. Preparation of 4-methoxyphenyl 4-allyloxybenzoate (MPAB)

To a 500-mL, 3-neck round bottom flask equipped with a reflux condenser, a drying tube and a magnetic stirrer, $12.00 \mathrm{~g}$ (0.0966 moles) of 4-methoxyphenol were transferred, followed by $150 \mathrm{~mL}$ of dry THF and $16 \mathrm{~mL}$ of dry pyridine. The mixture was kept under a nitrogen blanket and over a 2-hour period $19.00 \mathrm{~g}$ (0.0966 moles) of 4-(allyloxy)benzoyl chloride in $20 \mathrm{~mL}$ of dry pyridine were added through a dropping funnel. The mixture was stirred at room temperature for 16 hours and then heated at $60^{\circ} \mathrm{C}$ for two more hours. The reaction mixture was cooled to room temperature and $200 \mathrm{mil}$ of deionized water were added. The mixture was neutralized with $20 \%(\mathrm{v} / \mathrm{v}) \mathrm{HCl}$ and the organic material was extracted 3 times with diethyl ether. The solvent was removed by rotary evaporation and the product was dried under vacuum and weighed $(21.00 \mathrm{~g})$. The final product was recrystallized from ethanol twice yielding $76 \%$ of final product. m.p. 87.0-88.5 ${ }^{\circ} \mathrm{C}$. 


\section{Synthesis of dicyclopentadienyl platinum (II) chloride}

$1.0 \mathrm{~g}$ of chloroplatinic acid hydrate was dissolved in $2.2 \mathrm{~mL}$ of glacial acetic acid in a $10-\mathrm{mL}$ flask. The solution was diluted with $3.6 \mathrm{~mL}$ of water and then heated to $70^{\circ} \mathrm{C}$. $0.8 \mathrm{~mL}$ of dicyclopentadiene was then added to the reaction vessel and the mixture was stirred at $70^{\circ} \mathrm{C}$ for 24 hours. The crude product was collected by filtration, decolorized by charcoal and finally recrystallized twice from THF yielding $0.40 \mathrm{~g}(52 \%)$ of the final product [26].

\section{E. Preparation of hydrided silica}

1. Chlorination/reduction sequence

a. Chlorination of silica

$14.00 \mathrm{~g}$ of silica (approximately $8.85 \mathrm{mmols}$ of silanol, assuming $7.6 \mu \mathrm{mols}$ $\mathrm{OH} / \mathrm{m}^{2}$ ) were transferred to a $500-\mathrm{mL}$, 3-neck round bottom flask equipped with a magnetic stir bar and dried in a vacuum oven at $120^{\circ} \mathrm{C}$ overnight. Once cooled, 150 $\mathrm{mL}$ of freshly distilled dry toluene were added to the flask followed by $30 \mathrm{~mL}$ of thionyl chloride $\left(\mathrm{SOCl}_{2}\right)$ under a continuous stream of nitrogen. Chlorination was allowed to proceed under reflux for 48 hours, after which the reaction content was allowed to cool at room temperature and settle. The supernatant liquid was drained off under nitrogen environment and washed 6 times with 75-mL portions of dry 
toluene. The product was kept under about 50-mL of dry toluene before proceeding to the next step.

\section{b. Reduction of chlorinated silica}

A dry ice condenser was attached to the assembly to safely condense volatile

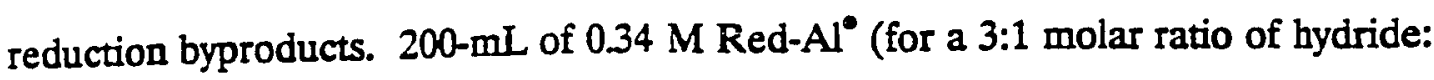
chloride assuming $100 \%$ conversion during chlorination) was added dropwise to the chlorinated silica suspension while stirring vigorously. The reaction was allowed to proceed for 24 hours under a gentle reflux upon addition of the reducing solution. The color of the silica turned from dark blue to beige. The solid was washed 5 times with 75-mL portions of dry toluene to remove excess of reducing agent. The crude product was then filtered through a ' $\mathrm{F}$ ' size fritted glass funnel, rinsed with $50 \mathrm{~mL}$ of diethyl ether 2 times and dried at room temperature for several hours. The product was then added slowly to a flask containing $100 \mathrm{~mL}$ of $0.5 \mathrm{M} \mathrm{HCl}$. The mixture was stirred for 10 minutes and allowed to settle down. The supernatant liquid was decanted and the process repeated 4 more times. The final product was successively washed with $50-\mathrm{mL}$ portions of water(2x), 1:1 v/v water/methanol(2x), methanol(2x), 1:1 v/v methanol/ether(2x), ether(2x). The final product was dried for several hours at room temperature and then in a vacuum oven at $120^{\circ} \mathrm{C}$ overnight. 2. Silanetriol condensation on silica

$1.50 \mathrm{~g}$. of silica (approximately $0.95 \mathrm{mmols}$ of silanol, assuming $7.6 \mathrm{\mu mol}$ $\mathrm{OH} / \mathrm{m}^{2}$ ) were transferred to a 25 -mL 3-neck round bottom flask equipped with a stir 
bar and dried in a vacuum oven at $120^{\circ} \mathrm{C}$ overnight. Once cooled, $35 \mathrm{~mL}$ of dioxane (dried over $\mathrm{CaH}_{2}$ ) were added to it, followed by $0.4 \mathrm{~mL}$ of concentrated $\mathrm{HCl}$ and $16.8 \mu \mathrm{L}$ of water. The content was heated to $80^{\circ} \mathrm{C}$. and $235 \mu \mathrm{L}$ of triethoxy silane (TES) dissolved in $10.00 \mathrm{~mL}$ of dioxane were transferred to the reaction mixture dropwise through a dropping funnel. The temperature of the contents was raised to $100{ }^{\circ} \mathrm{C}$ after the addition was over and refluxed for 1 hour. The reaction mixture was then centrifuged and washed twice with $10-\mathrm{mL}$ portions of dioxane/water $(1: 1 \mathrm{v} / \mathrm{v}), 10 \mathrm{~mL}$ of THF twice and $10-\mathrm{mL}$ of ether twice. Ether was evaporated at room temperature and the hydrided silica was dried in vacuum at $120^{\circ} \mathrm{C}$ overnight.

\section{F. Hydrosilation of MPAB on hydrided silica}

To a 15-mL, 3-neck round bottom flask, $0.215 \mathrm{~g}$ (3.52mmols) of 4-methoxy-phenyl 4-allyloxybenzoate (MPAB) were added. A bent glass tube containing 0.500 $\mathrm{g}$ of dry hydrided silica was attached to it. $7.5 \mathrm{~mL}$ of toluene (dried over $\mathrm{CaH}_{2}$ ) were transferred to the flask followed by $60.4 \mu \mathrm{L}$ of $2.5 \mathrm{mM}$ platinum (II) catalyst solution in $\mathrm{CHCl}_{3}$. After flushing the flask with nitrogen, the contents in the flask was heated to and maintained at $70^{\circ} \mathrm{C}$ for about one hour after which the hydrided silica was added to the reaction mixture slowly by rotating the bent tube. The temperature was increased and the reaction was allowed to proceed at $100 \pm 2{ }^{\circ} \mathrm{C}$ for 48 hours. Once the reaction was over, the product was washed 4 times with $5-\mathrm{mL}$ portions of dry 
toluene followed by $5-\mathrm{mL}$ portions of $\mathrm{CH}_{2} \mathrm{Cl}_{2}$ (twice) and $5-\mathrm{mL}$ portions of ether (twice). The product was dried under vacuum at room temperature for 24 hours.

\section{G. Hydrosilation of cholesteryl 10-undecenoate on hydrided silica}

To a 15 -mL, 3-neck round bottom flask, $0.418 \mathrm{~g}(0.756 \mathrm{mmols})$ of cholesteryl 10-undecenoate were added. A bent glass tube containing $0.500 \mathrm{~g}$ of dry hydrided silica was attached to it. $7.5-\mathrm{mL}$ of toluene (dried over $\mathrm{CaH}_{2}$ ) was transferred to the flask followed by $60.4 \mu \mathrm{L}$ of $2.5 \mathrm{mM}$ of platinum(II) catalyst solution in $\mathrm{CHCl}_{3}$. After flushing the flask with nitrogen, the contents in the flask were heated to and maintained at $70{ }^{\circ} \mathrm{C}$ for about one hour after which the hydrided silica was added to the reaction mixture slowly by rotating the bent tube. The temperature was increased and the reaction was allowed to proceed at $100 \pm 2{ }^{\circ} \mathrm{C}$ for 48 hours. Once the reaction was over, the product was washed 4 times with 5-mL portions of dry toluene followed by $5-\mathrm{mL}$ portions of $\mathrm{CH}_{2} \mathrm{Cl}_{2}$ (twice) and 5 - $\mathrm{mL}$ portions of ether (twice). The product was dried under vacuum at room temperature for 24 hours. 


\section{CHAPTER III}

\section{RESULTS AND DISCUSSION}

The principal goal of this work was to explore the feasibility of bonding the two aforementioned compounds to a silica support bearing surface $\mathrm{Si}-\mathrm{H}$ groups according to the reaction

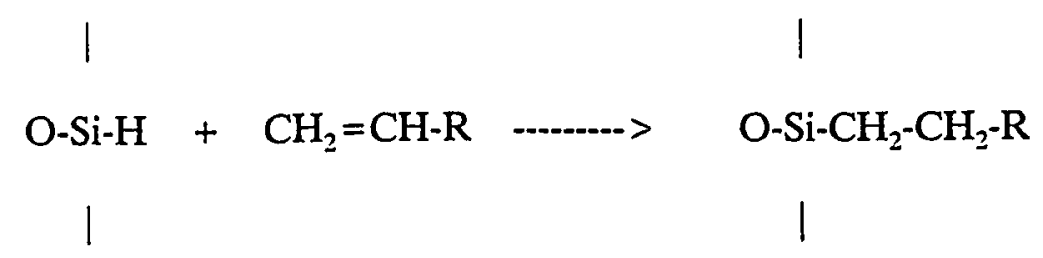

\section{A. Surface coverage}

Surface coverage, $\alpha_{R}$, of the organic substrate was obtained from the carbon percent of the bonded material and BET specific surface area of the hydrided silica substrate before bonding. Assuming a surface monolayer of organic compounds formed on the surface, the equation proposed by Berendsen and De Galan [28] was used

$$
\alpha_{R}\left(\mu \mathrm{mol} / \mathrm{m}^{2}\right)=\frac{10^{6} P_{c}}{\left(10^{2} M_{c} \Omega_{c}-P_{c} M_{R}\right) S}
$$


where $\mathrm{P}_{\mathrm{c}}$ is the corrected carbon percentage (after subtracting carbon present in the hydrided silica); $\mathrm{n}_{\mathrm{c}}$ represents the number of carbon atoms in the bonded organic group (in this case, phenyl ester and cholesteryl ester); $S$ is the BET specific surface area $\left(\mathrm{m}^{2} / \mathrm{g}\right)$ of the intermediate hydrided surface; $\mathrm{M}_{\mathrm{R}}$ is the molecular weight of phenyl ester and cholesteryl ester, as may be the case, and $M_{c}$ is the atomic weight of carbon. Since this is an addition reaction, the atomic weight of the hydrogen does not need to be subtracted [29].

\section{B. Effect of catalyst on hydrosilation}

The hydrosilation of liquid crystal on modified silica was initiated with two different catalytic systems

1) Dicyclopentadienyl platinum (II) chloride, and

2) Platinum hexachloride (hexachloroplatinic acid)

The effect of catalyst on surface coverage is shown in Figure 6 and Table 1. Under the same reaction conditions the nature of the catalyst seems to affect the surface hydrosilation of MPAB while being irrelevant to that of cholesteryl 10undecenoate. Surface coverage of MPAB on silica is $1.005 \mu \mathrm{mol} / \mathrm{m}^{2}$ when dicyclopentadienyl platinum (II) chloride is used as catalyst, compared to 0.721 $\mu \mathrm{mol} / \mathrm{m}^{2}$ when hexachloroplatinic acid is used instead. Surface coverage of cholesteryl 10 -undecenoate is more or less the same $\left(0.252 \mu \mathrm{mol} / \mathrm{m}^{2}\right.$ vs 0.241 $\left.\mu \mathrm{mol} / \mathrm{m}^{2}\right)$ in both catalytic systems. 


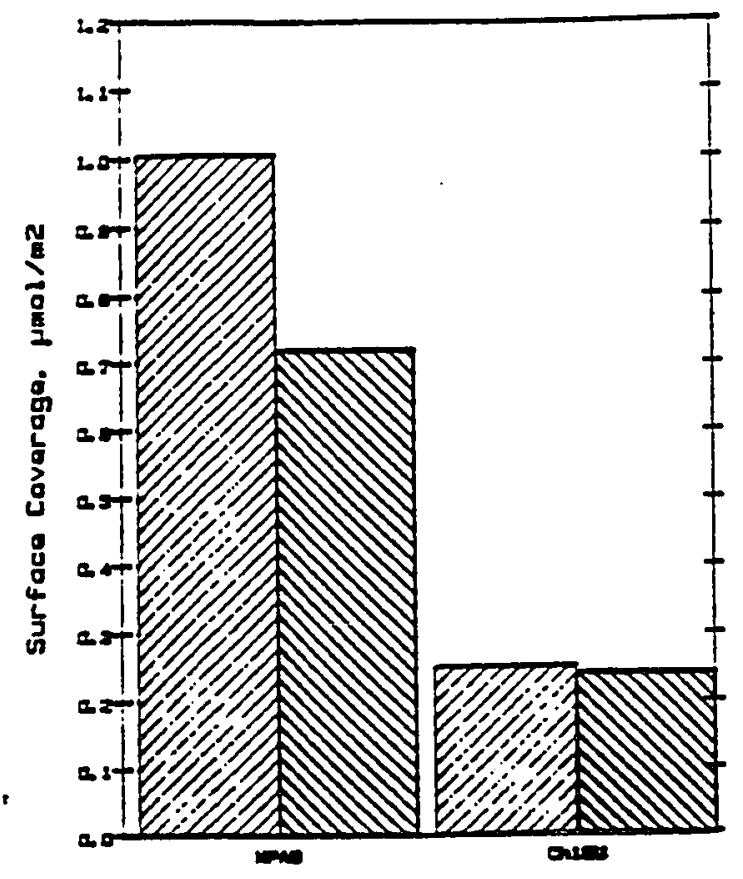

Figure 6. Effect of catahyst on surface coverage platinum (II) chloride and $\mathrm{SIS}$ herachloroplatinic acid.

TABLE 1. Hydrosilation of liquid crystals on hydrided silica using two different catalytic systems.

\begin{tabular}{|c|c|c|c|}
\hline Liquid crystal & Catalyst & $\begin{array}{c}\% \text { Corrected } \\
\text { carbon }\end{array}$ & $\begin{array}{c}\text { Surface coverage } \\
\mu \mathrm{mol} / \mathrm{m}^{2}\end{array}$ \\
\hline 4-Methoxyphenyl 4-allyloxybenzoate & $\mathrm{DCPP}$ & 1.62 & 1.01 \\
\hline 4-Methoxyphenyl 4-allyloxybenzoate & $\mathrm{H}_{2} \mathrm{PtCl}_{8} \mathrm{xH}_{2} \mathrm{O}$ & 1.17 & 0.72 \\
\hline Cholesteryl 10-undecenoate & $\mathrm{DCPP}$ & 0.92 & 0.25 \\
\hline Cholesteryl 10-undecenoate & $\mathrm{H}_{2} \mathrm{PtCl}_{6} \times \mathrm{XH}_{2} \mathrm{O}$ & 0.88 & 0.24 \\
\hline
\end{tabular}


As expected, both cataiytic systems gave the same hydrosilylation product for MPAB and cholesteryl 10-undecenoate. Partial DRIFT spectrum (Figure 7) shows typical comparison of cholesteryl 10-undecenoate bonded to silica using two different kinds of catalyst. Identical peaks in $3170-2690 \mathrm{~cm}^{-1}$ region shows aliphatic carbon bonded to silica. In addition to superior surface coverage, dicyclopentadienyl platinum (II) chloride is easier to handle, we decided to use it for the future work.

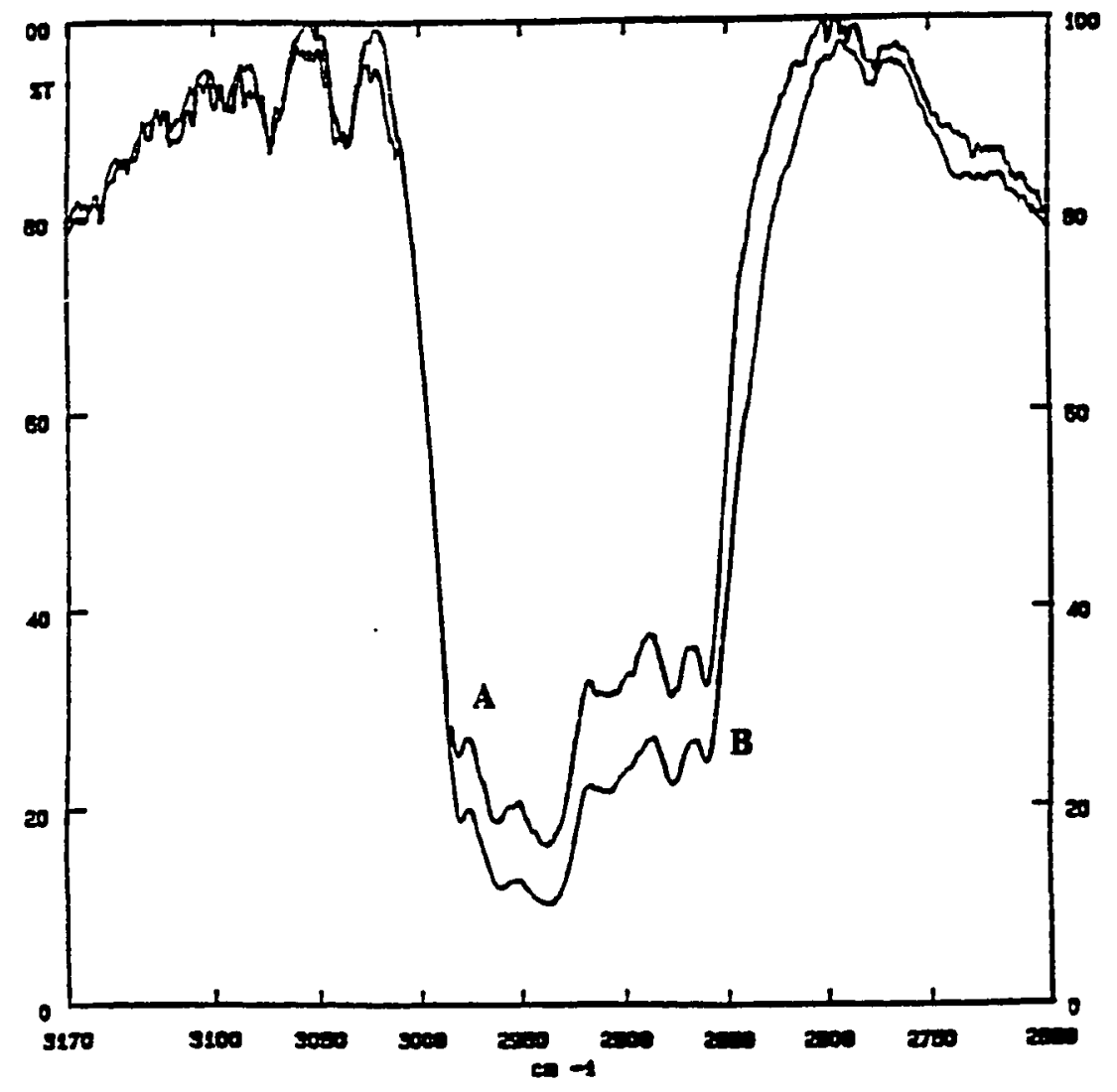

Figure 7. Partial DRIFT spectral comparison of cholesteryl 10-undecenoate bonded to silica: A) bonded phase prepared by using hexachloroplatinic acid, and $B$ ) by using dicyclopentadienyl platioum (II) chloride 
C. Effect of time on surface coverage

In order to explore the reaction's completeness, we also monitored the reaction progress with time for 96 hours by taking an aliquot at every 12 -hour interval. After solvent removal and drying, the bonded silica sample was analyzed for carbon content. The graphic presentation of results is given in Figure 8 .

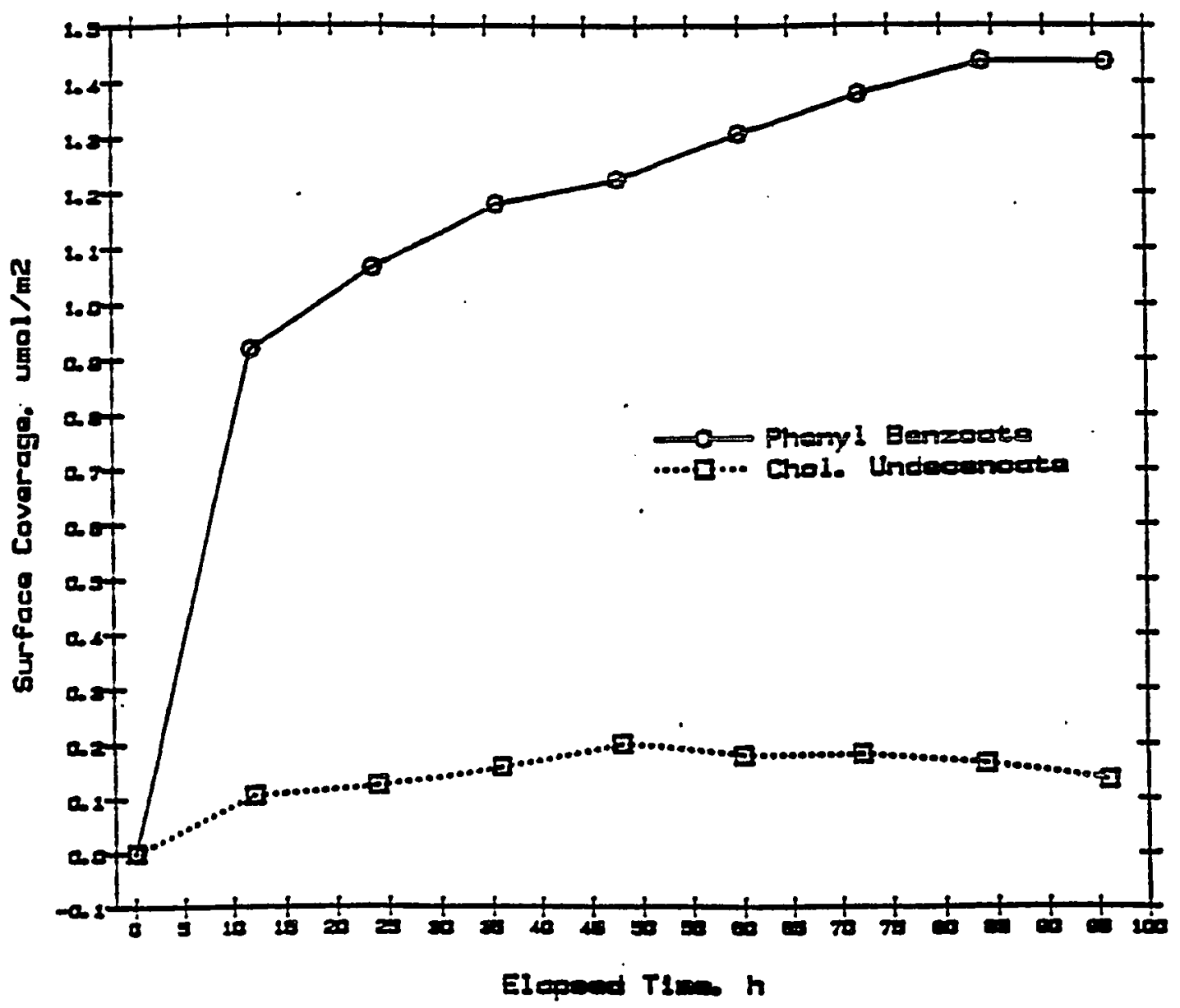

Figure 8. Surface coverage as a function of time. 
Carbon analysis results are given in Tables 2 and 3 . After about 60 hours the reaction seems to level off.

TABLE 2. Surface coverage as a function of reaction time. Cholesteryl 10-undecenoate on hydrided silica.

\begin{tabular}{||c|c|c||}
\hline \hline Elapsed time in hr. & \% Corrected carbon & Surface coverage $\mu \mathrm{mol} / \mathrm{m}^{2}$ \\
\hline 0 & 0.00 & 0.00 \\
\hline 12 & 0.39 & 0.11 \\
\hline 24 & 0.46 & 0.13 \\
\hline 36 & 0.58 & 0.16 \\
\hline 48 & 0.73 & 0.20 \\
\hline 60 & 0.65 & 0.18 \\
\hline 72 & 0.66 & 0.18 \\
\hline 84 & 0.60 & 0.16 \\
\hline 96 & 0.49 & 0.13 \\
\hline
\end{tabular}

$T A B L E$ 3. Surface coverage as a function of reaction time. 4-Methoxyphenyl 4-allyloxybenzoate on hydrided silica.

\begin{tabular}{|c|c|c|}
\hline Elapsed time in hr. & \% Corrected carbon & Surface coverage $\mu \mathrm{mol} / \mathrm{m}^{2}$ \\
\hline 0 & 0.00 & 0.00 \\
\hline 12 & 1.48 & 0.92 \\
\hline 24 & 1.71 & 1.06 \\
\hline 36 & 1.89 & 1.18 \\
\hline 48 & 1.96 & 1.22 \\
\hline 60 & 2.09 & 1.31 \\
\hline 72 & 2.20 & 1.38 \\
\hline 84 & 2.29 & 1.44 \\
\hline 96 & 2.29 & 1.44 \\
\hline
\end{tabular}




\section{Effect of hydride intermediate on surface coverage}

In this work two synthetic procedures were used to attach $\mathrm{SiH}$ species on the silica surface: a chlorination/reduction procedure (Chapter II, p. 23) and a silanetriol condensation process (Chapter II, p. 24). Figure 9 depicts a comparison of the two intermediate substrates with respect to the surface coverage of the two esters. Under the same reaction conditions, the silane intermediate produced by the chlorination/reduction technique resulted in lower surface coverages for both esters (Table 4). Silanetriol condensation process is believed to form a surface monolayer on silica material, protruding silane groups on the surface. As a result more silane groups are available for bonding. In addition to providing superior coverages, the silane deposition substrate is easier and safer to prepare, this intermediate was used in the remainder of the work. Moreover, the procedure used to prepare this substrate is free from any metal contamination (namely aluminum chemisorption) which normally accompanies the chlorination/reduction method. ESCA scans performed on hydrided materials did not show any metal contamination, in agreement with previously reported results [8]. 
TABLE 4. Hydrided vs silane deposited intermediate.

\begin{tabular}{||c|c|c||}
\hline Sample ID & $\begin{array}{c}\text { \% Corrected } \\
\text { carbon }\end{array}$ & $\begin{array}{c}\text { Surface coverage } \\
\mu \mathrm{mol} / \mathrm{m}^{2}\end{array}$ \\
\hline MPAB on hydrided silica & 1.62 & 1.01 \\
\hline MPAB on silane deposited silica & 2.11 & 1.32 \\
\hline Chol. ester on hydrided silica & 0.92 & 0.25 \\
\hline Chol. ester on silane deposited silica & 1.17 & 0.32 \\
\hline
\end{tabular}

Figure 9 represents the effect of intermediate on the surface coverage.

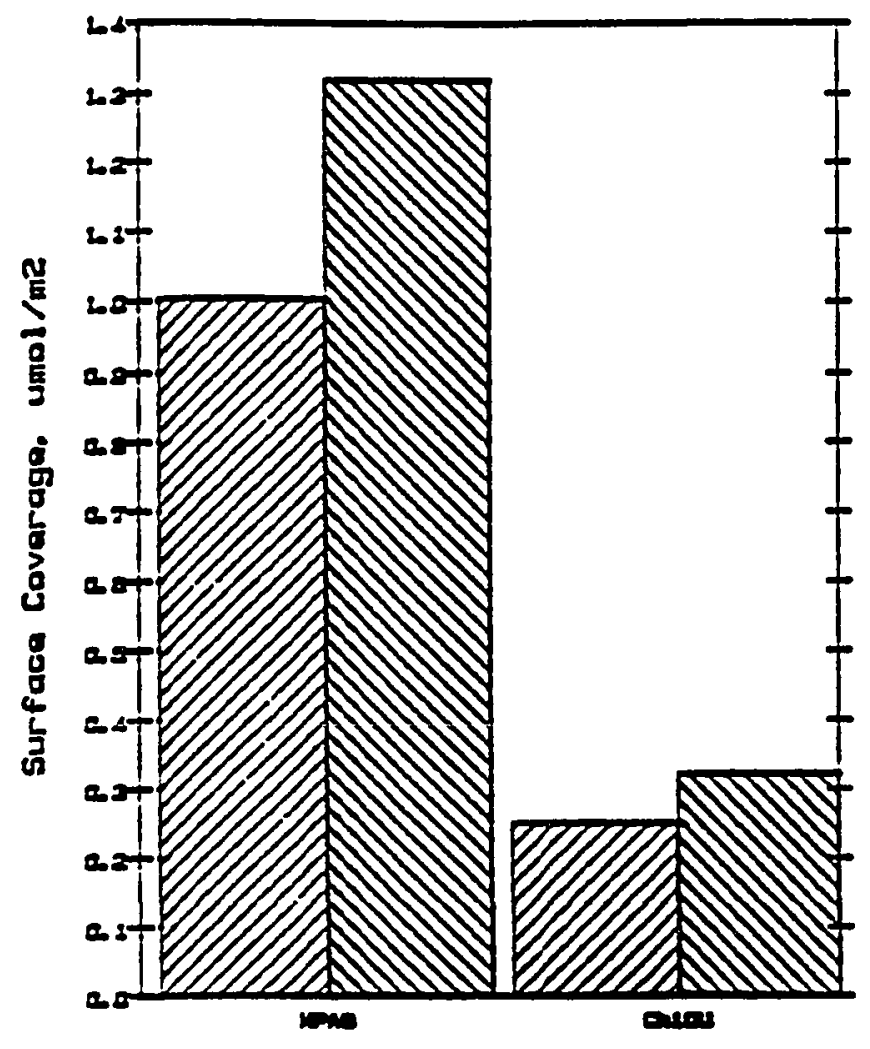

Figure 9. Effect of intermediate on sufface coverage 2019 Chlorination/reduction product and silanetriol condensation product. 


\section{E. Spectroscopic evaluation}

Infrared spectroscopy is a very powerful technique for studying surface chemistry. It has proved itself to be an important tool in the investigation of chemical reactions between the surface's active sites and the anchored species. Silica has been the material of preference in chromatography because of its large surface area and relatively high mechanical strength. Diffuse reflectance infrared fourier transform (DRIFT) spectroscopy provides a means of readily identifying the surface species and monitoring chemical changes. We have used silica as starting material. Silica surfaces are usually covered with a layer of Si-OH groups and adsorbed $\mathrm{H}_{2} \mathrm{O}$. When the hydride intermediate is formed, extensive removal of Si-OH groups takes place and Si-H species appear on the surface. Finally, when olefins undergo addition, C-H as well as other groups show up, with concomitant depletion of Si-H groups. DRIFT thus provides a rapid qualitative evaluation of the surface after chemical modification.

\section{Native silica}

A typical DRIFT spectrum of native silica is shown in Figure 10. A sharp band at $3750 \mathrm{~cm}^{-1}$ is assigned to "isolated" (i.e., not $\mathrm{H}$-bonded) $\mathrm{OH}$ groups. Absorption centered in the $3500-3400 \mathrm{~cm}^{-1}$ region is attributed to strongly $\mathrm{H}$-bonded OH groups and/or adsorbed $O H$ groups. In addition, a band near $1630 \mathrm{~cm}^{-1}$ is also assigned to $\mathrm{H}_{2} \mathrm{O}$. Strong absorption bands at 1200 and $1100 \mathrm{~cm}^{-1}$ are attributable to 
fundamental silicon-oxygen vibrations and are observed in all forms of silicon dioxide. A band near $870 \mathrm{~cm}^{-1}$ has been assigned to deformation of surface $\mathrm{OH}$ groups (Figure 10).

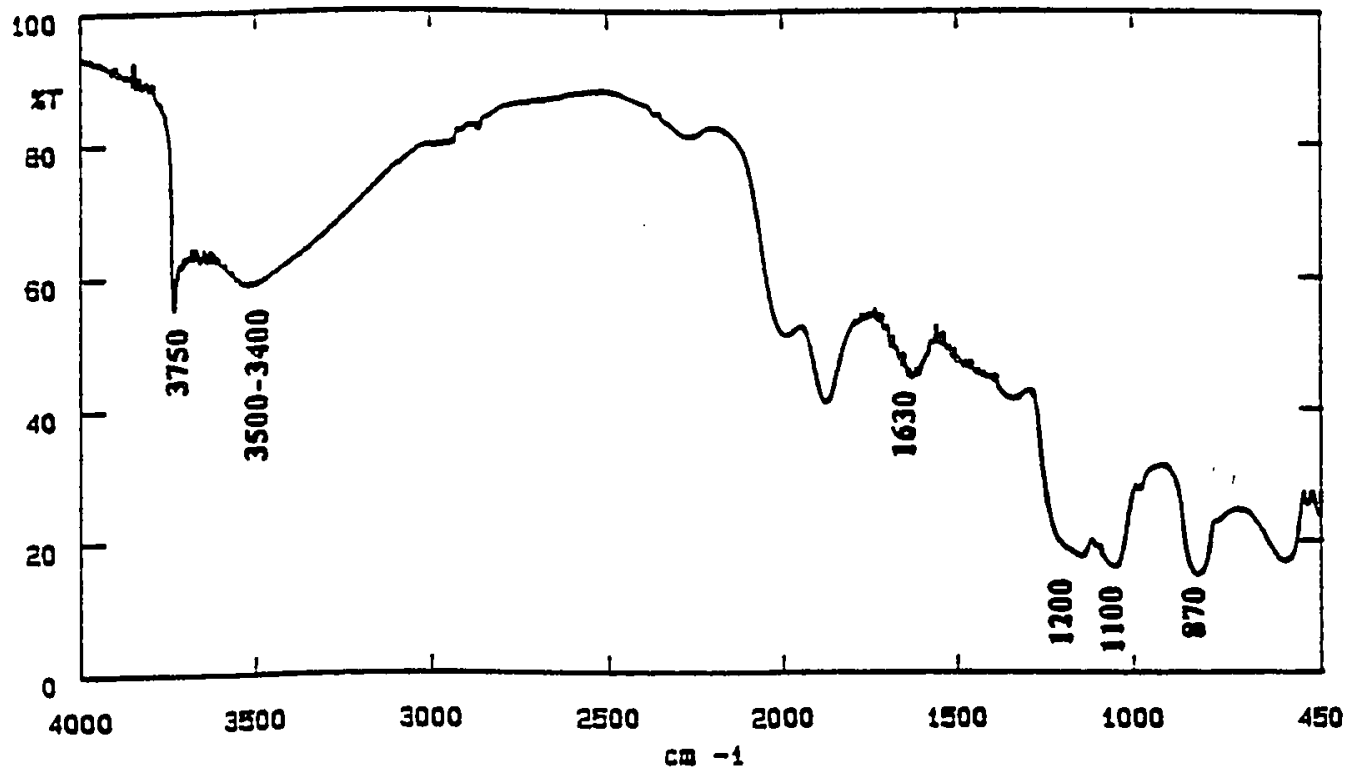

Figure 10. DRIFT spectrum of native silica.

\section{Hydride intermediate}

The Si-H stretching vibration is one of the most characteristic IR frequency for silane compounds. It gives a strong band in the $2300-2100 \mathrm{~cm}^{-1}$ region where there is very little interference from other bands. It has been shown that the position of Si-H stretching frequency is strongly dependent on the inductive power of the other groups on the silicon atom, the more electron withdrawing groups causing shift 
toward higher frequencies. When chlorinated silica was reduced with an excess of Red-AL, a sharp peak was produced at about $2260 \mathrm{~cm}^{-1}$. This is a characteristic peak for Si-H stretching (Figure 11). Additionally, the weak bending at 910 and 760 $\mathrm{cm}^{-1}$ is also present. As expected, these peaks are equivalent to those reported by Sandoval and Pesek [8] while reducing chlorinated silica with $\mathrm{LiAlH}_{3}$.

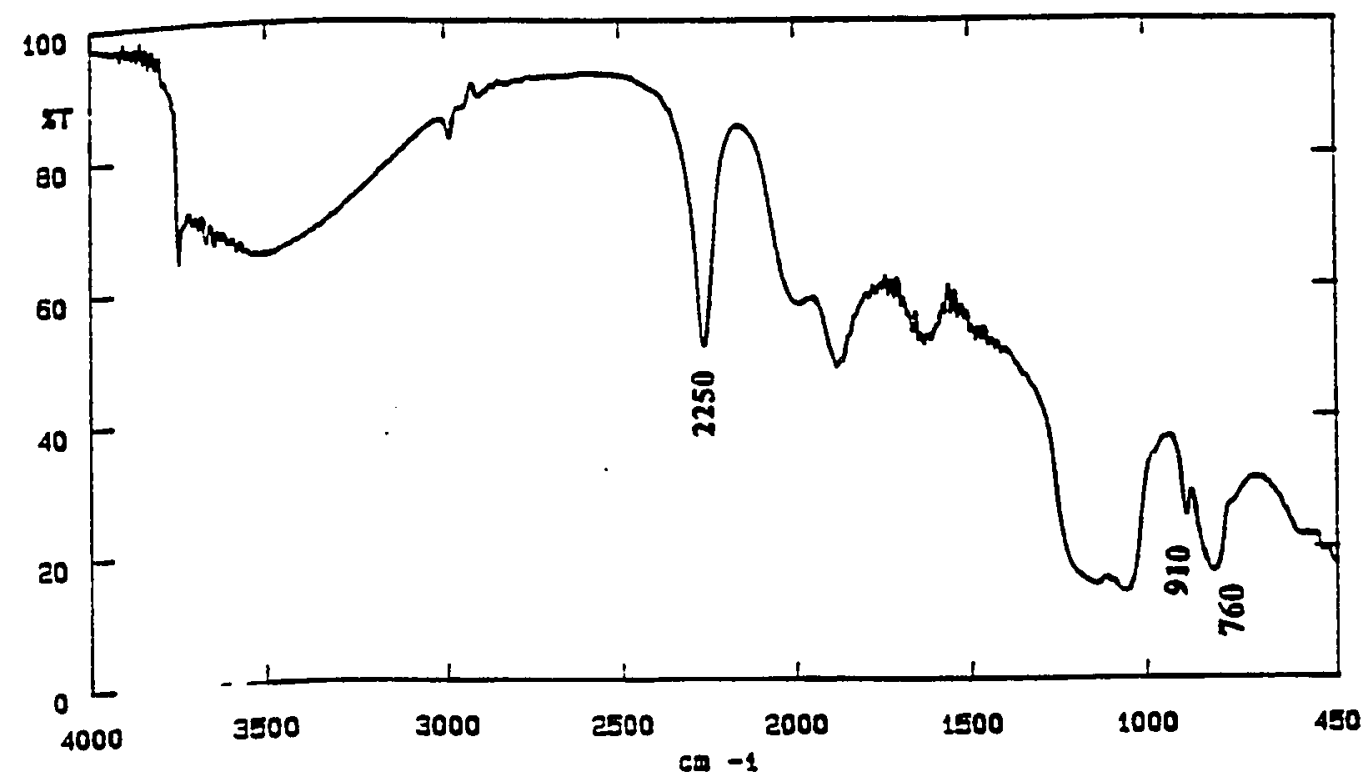

Figure 11. DRIFT spectrum of hydrided silica.

3. 4-Methoxyphenyl 4-allyloxybenzoate (MPAB)

MPAB was synthesized using the technique described by Laub and coworkers [26]. The comparatively large structure of MPAB gives a complex IR spectrum and overlapping of certain peaks is observed (Figure 12). A peak at $3082 \mathrm{~cm}^{-1}$ represents the allylic C-H stretching. A peak at $2942 \mathrm{~cm}^{-1}$ indicates $\mathrm{CH}_{3}$ stretching vibrations 
from methoxy group, which is further confirmed by an ester stretch C-O at $1510 \mathrm{~cm}^{-1}$. Peaks in the region of $3050-3000 \mathrm{~cm}^{-1}$ represent aromatic stretching and peaks in the region of 3000-2850 $\mathrm{cm}^{-1}$ represent aliphatic $\mathrm{C}-\mathrm{H}$ stretching. A sharp peak at 1738 $\mathrm{cm}^{-1}$ is attributed to the carbonyl $(\mathrm{C}=\mathrm{O})$ bond. Peaks at $1635 \mathrm{~cm}^{-1}$ and $1260 \mathrm{~cm}^{-1}$ represent $\mathrm{C}-\mathrm{H}$ bending (Figure 12).

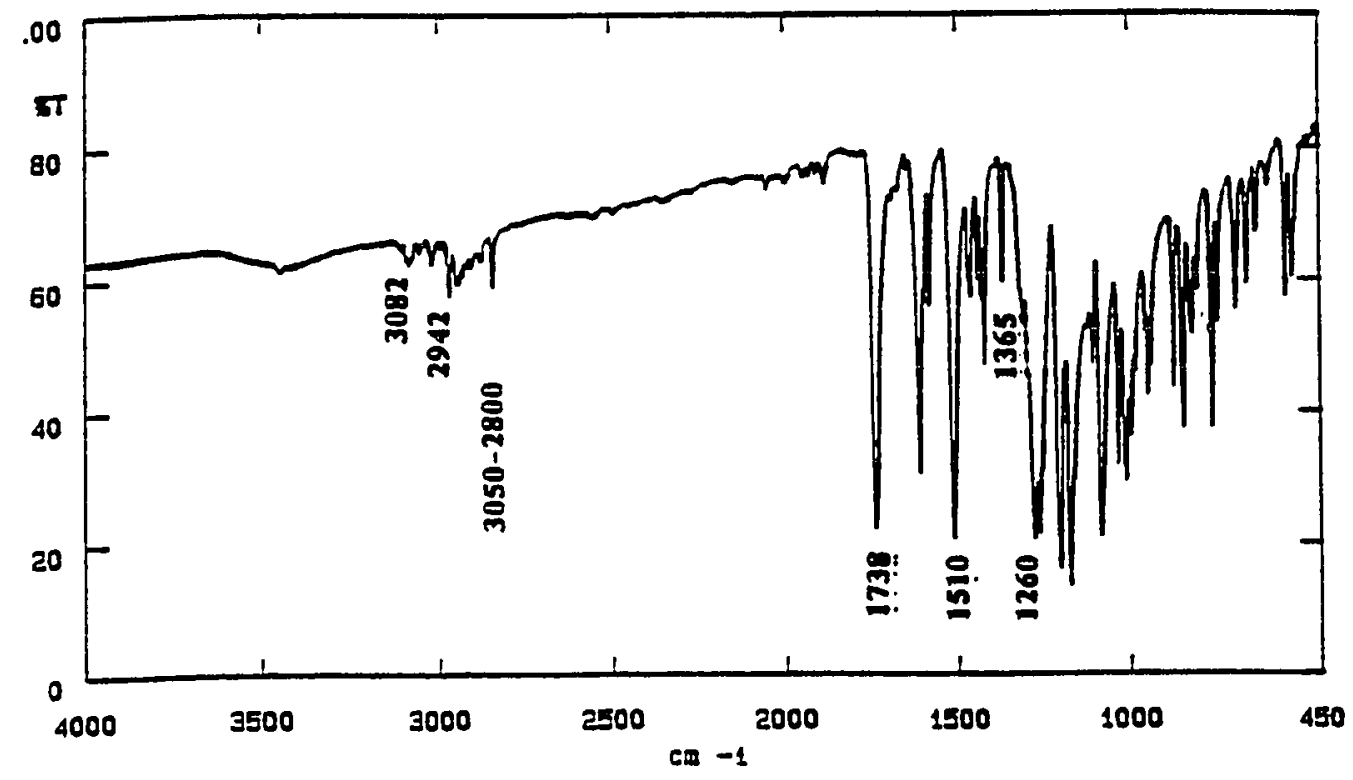

Figure 12. DRIFT spectrum of 4-methoxyphenyl 4-allyloxybenzoate.

\section{Cholesteryl 10-undecenoate}

Cholesteryl 10 -undecenoate is a commercially available product. The DRIFT spectrum of this material is shown in Figure 13. The weak band at $3080 \mathrm{~cm}^{-1}$ indicates the $\mathrm{C}-\mathrm{H}$ stretching frequency at the terminal double bond. The strong band in the region of $2930-2850 \mathrm{~cm}^{-1}$ represents aliphatic C-H stretching. A sharp peak 
at $1733 \mathrm{~cm}^{-1}$ indicates the presence of carbonyl $(C=0)$ group. Another sharp peak at $1468 \mathrm{~cm}^{-1}$ reveals a $\mathrm{C}-\mathrm{H}$ bending frequency. A peak at $1173 \mathrm{~cm}^{-1}$ comes from single $\mathrm{C}-\mathrm{O}$ stretch (Figure 13).

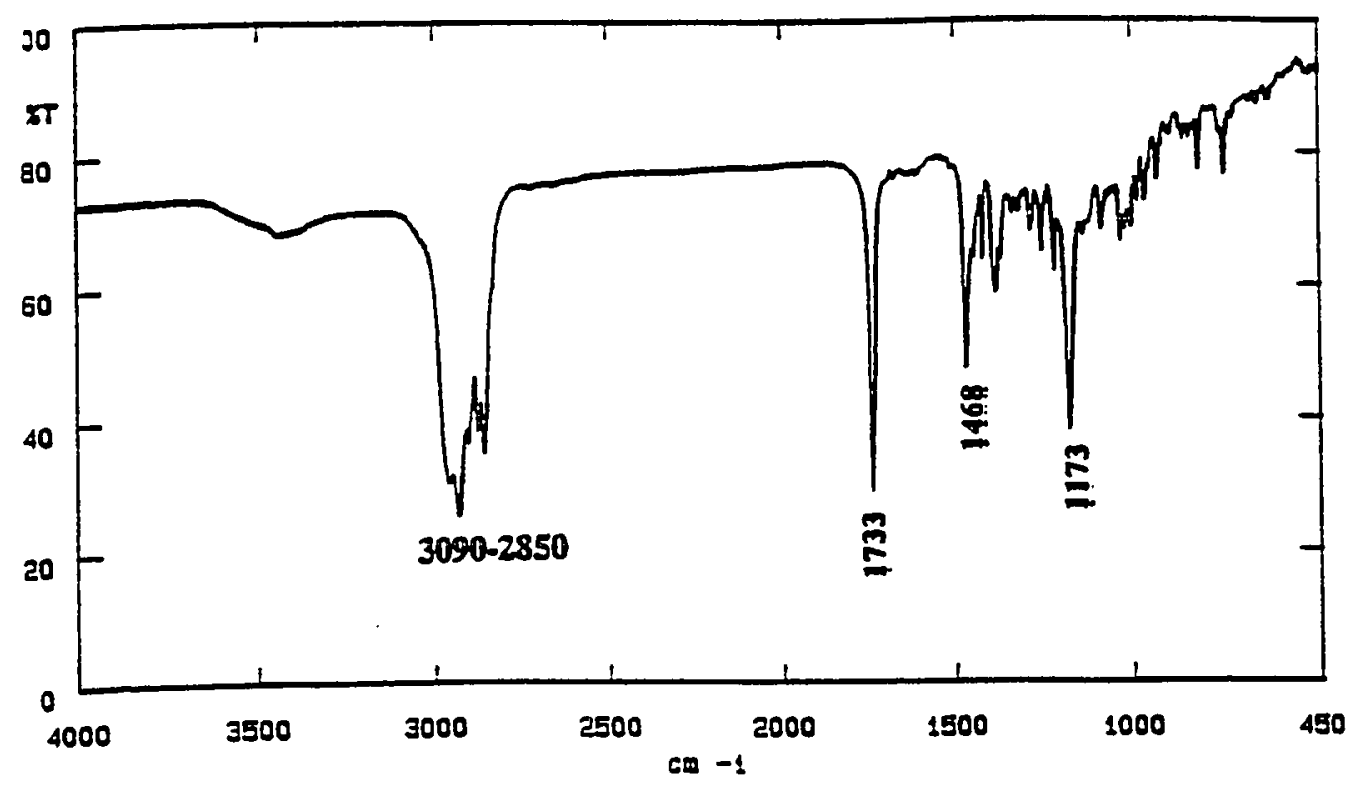

Figure 13. DRIFT spectrum of cholesteryl 10-undecenoate.

Figure 14 consists of a total of four spectra for comparison purposes. A and C are the spectra of two different batches of native silica. Spectrum B and spectrum $D$ are similar, although different modification processes were used. B represents the product prepared by chlorination/reduction technique using Red-Al reducing agent (Chapter II, p. 23), while spectrum D shows the product of silanetriol condensation (Chapter II, p. 24). As expected, spectrum $B$ and D are similar, indicating the same hydrided product. As mentioned earlier, the silanetriol condensation process is easy, 
fast and has less stringent reaction conditions. We decided to use this process with triethoxysilane as the hydrosilane material.

In Figure 15, Spectrum A represents silica reduced via chlorination/reduction technique. A distinct peak at $2260 \mathrm{~cm}^{-1}$ appears due to $\mathrm{Si}-\mathrm{H}$ stretching vibrations. $B$ is the spectrum obtained after bonding phenyl ester (MPAB in this case) to the reduced silica. The peak intensity at $2260 \mathrm{~cm}^{-1}$ has decreased. At the same time several peaks are emerging in the $3150-2750 \mathrm{~cm}^{-1}$ region, indicating aromatic and aliphatic C-H stretching. Carbon analysis supports the hypothesis that MPAB has been successfully bonded to the silica.

Figure 16 follows the same argument, except $A$ is the spectrum of the TES deposited silanized product instead of chlorination/reduction product. Again a decrease in the Si-H stretching frequency is observed and several peaks in 3150-2750 $\mathrm{cm}^{-1}$ region have appeared. 
41

Figure 14. Partial DRIFT spectra of native silica and hydrided intermediates: $A$ and $C$ are the spectra of native silica from two different batches. $B$ and $D$ are hydride intermediates, respectively.

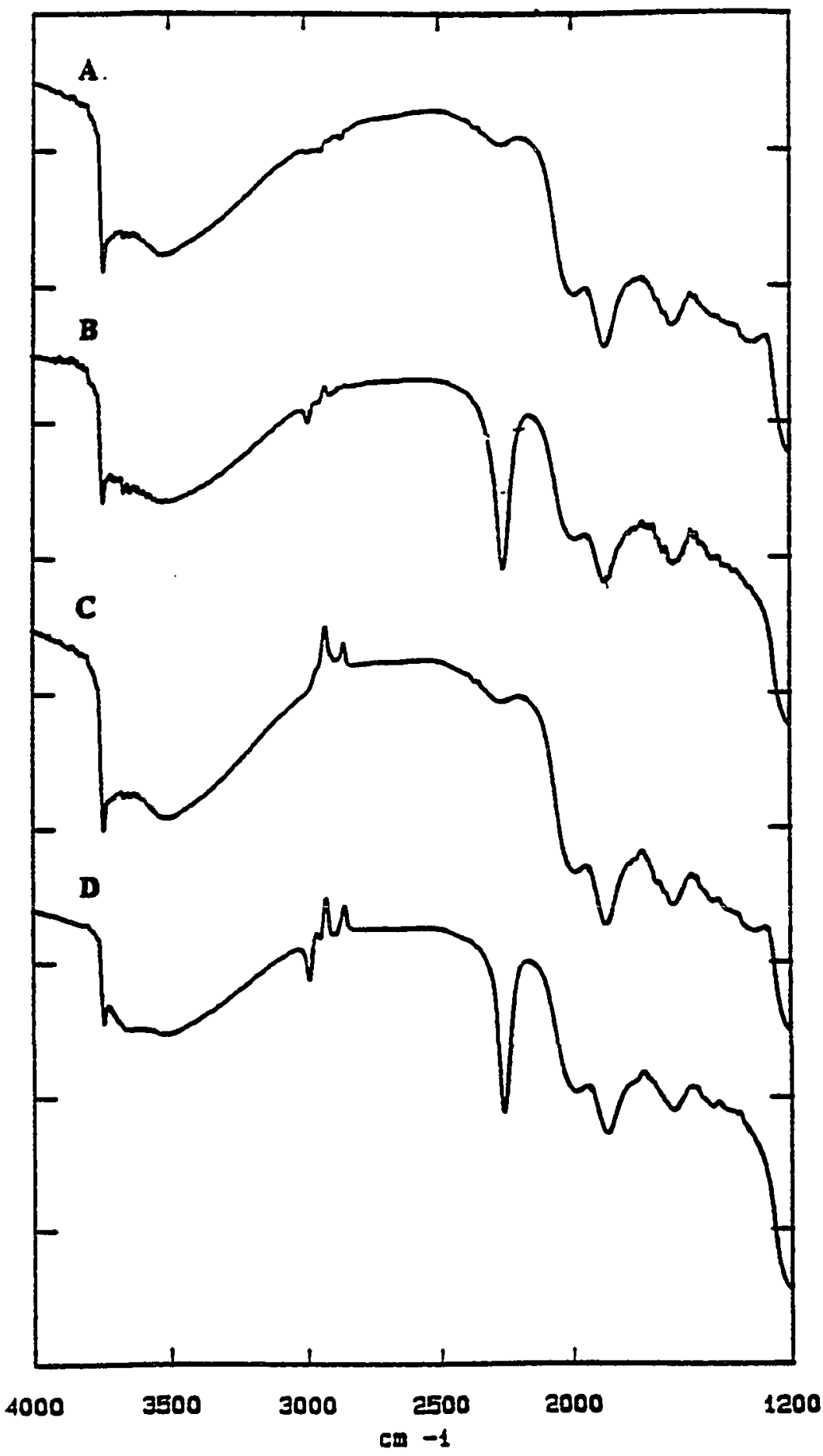


Figure 15. Partial DRIFT spectrum of silane intermediate prepared by chlorination/reduction technique $(A)$, and $(B)$ after bonding with 4-methoxyphenyl 4-allyloxybenzoate.

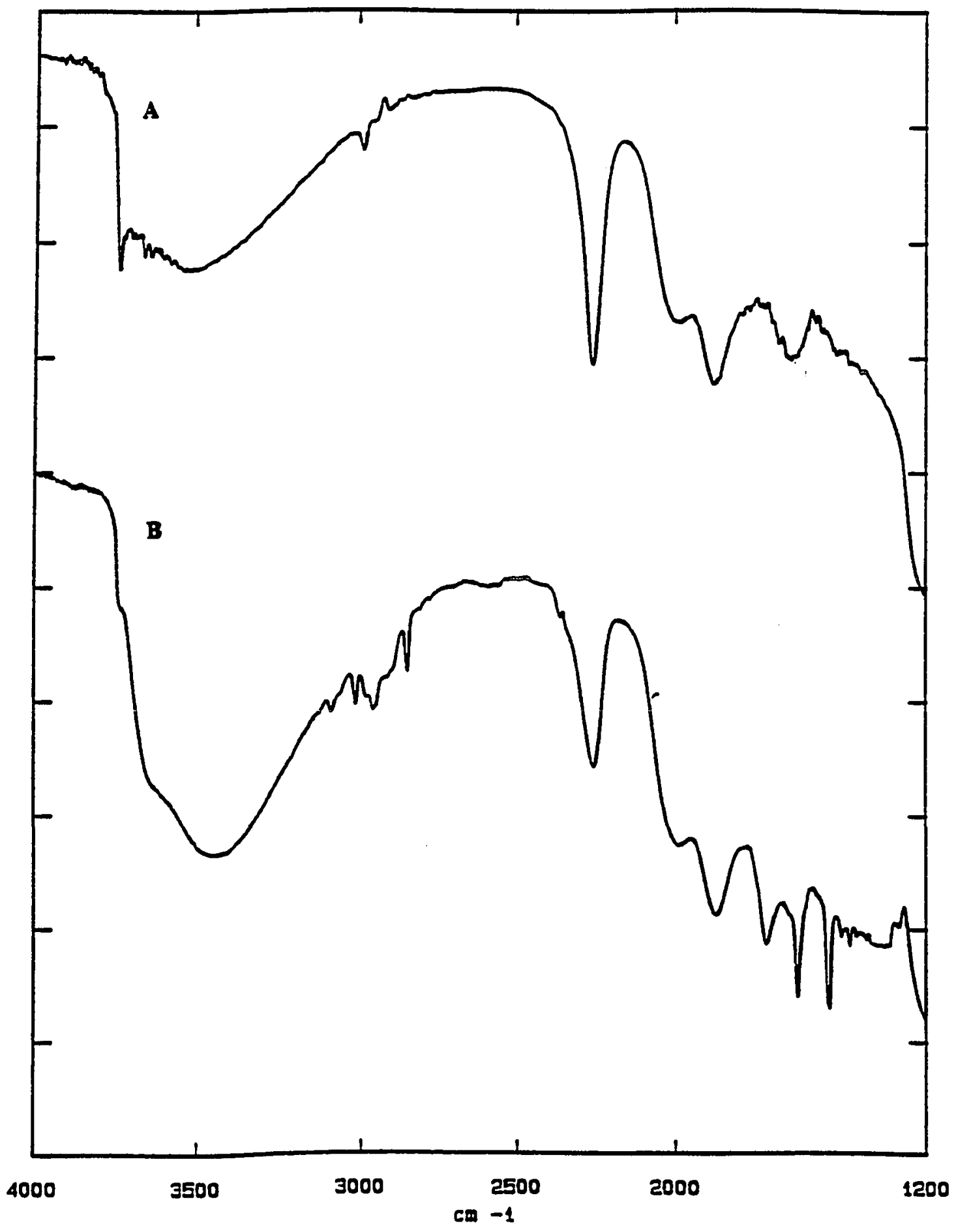


Figure 16. Partial DRIFT spectrum of silane intermediate prepared by silanetriol condensation technique $(A)$, and $(B)$ after bonding with 4-methoxyphenyl 4-allyloxybenzoate.

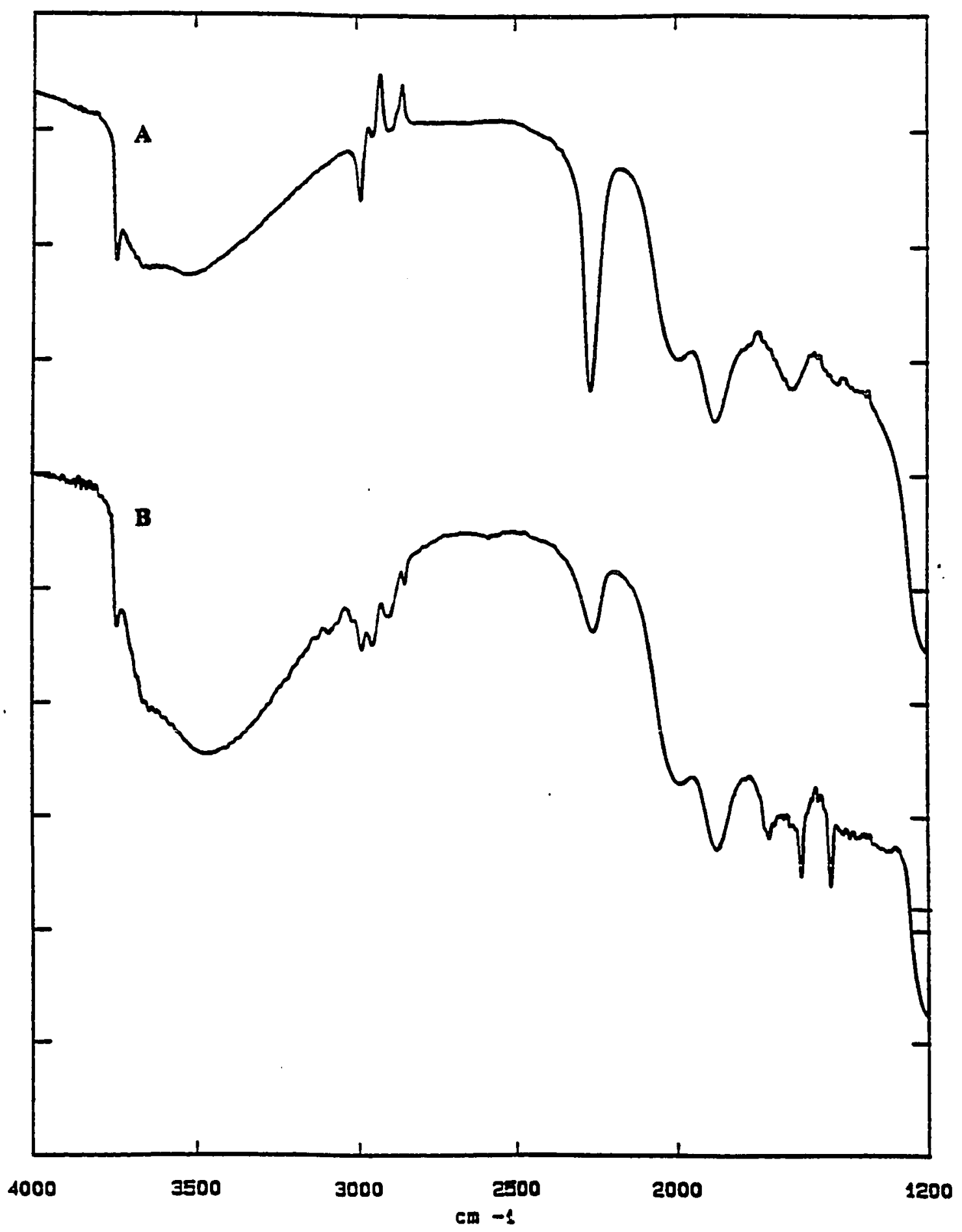


Figure 17 compares two hydrosilated products of 4-methoxyphenyl 4-allyloxy-benzoate (MPAB) on hydrided silica. Spectrum A was obtained by subtracting the spectrum of reduced silica prepared by the chlorination/reduction process and spectrum $B$ was the result of subtraction of silina modified by the TES deposition process from the hydrosilation product. Spectrum A and spectrum B are similar, indicating the same hydrosilation product. This information is important since it confirms the fact that the silane prepared by silane coupling of TES, which is easier and faster to prepare, can be used instead of the one prepared by the chlorination/reduction process.

Figure 18 shows two spectra. A represents the spectrum of hydrided silica prepared by chlorination/reduction process and B is the hydrosilation product of cholesteryl 10-undecenoate on hydrided silica. A decrease in the band frequency at $2260 \mathrm{~cm}^{-1}$ and the appearence of several new peaks in the $3150-2750 \mathrm{~cm}^{-1}$ region indicates successful bonding on silica.

Figure 19 follows the same argument except the hydrided silica in this case is the TES deposited product prepared by silanetriol condensation technique. A closer comparison of spectra B of Figure 18 with spectra B of Figure 19 reveals the "fact" that both the hydrosilanized products are the same, except that the product prepared from the hydride intermediate via silanetriol condensation (B) shows an extra peak at $3000 \mathrm{~cm}^{-1}$ (Figure 20). No explanation can be given at this time for the occurrence 
of this peak.

Figure 17. Partial DRIFT spectra of hydrided silica bonded with 4-methoxyphenyl 4-allyloxybenzoate: $(A)$ in hydride intermediate prepared via chlorination/reduction technique, and $(B)$ in hydride intermediate prepared by silanetriol condensation process.

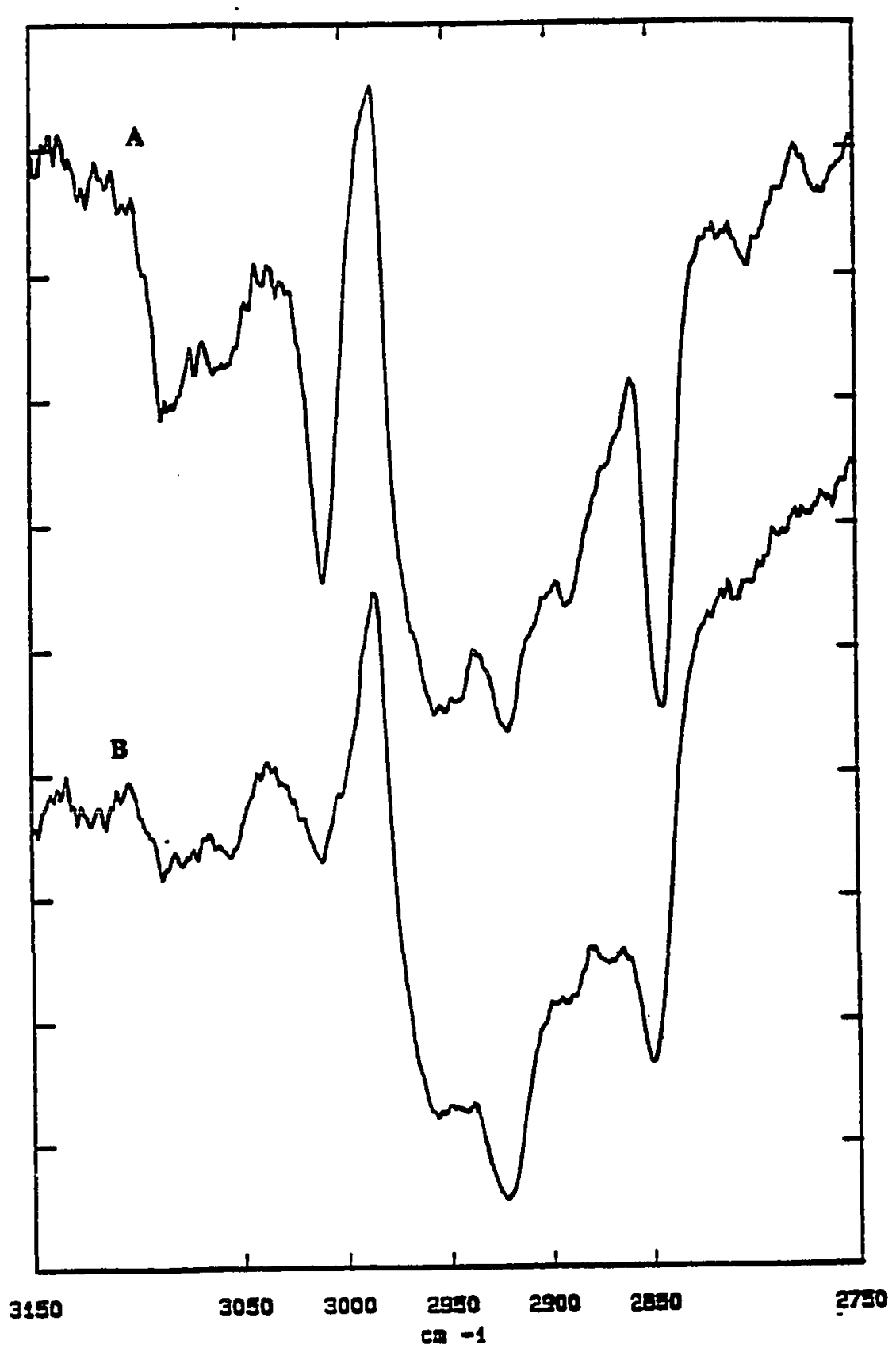


Figure 18. Partial DRIFT spectrum of silane intermediate prepared by chlorination/reduction technique $(A)$, and $(B)$ after bonding with cholesteryl 10-undecenoate.

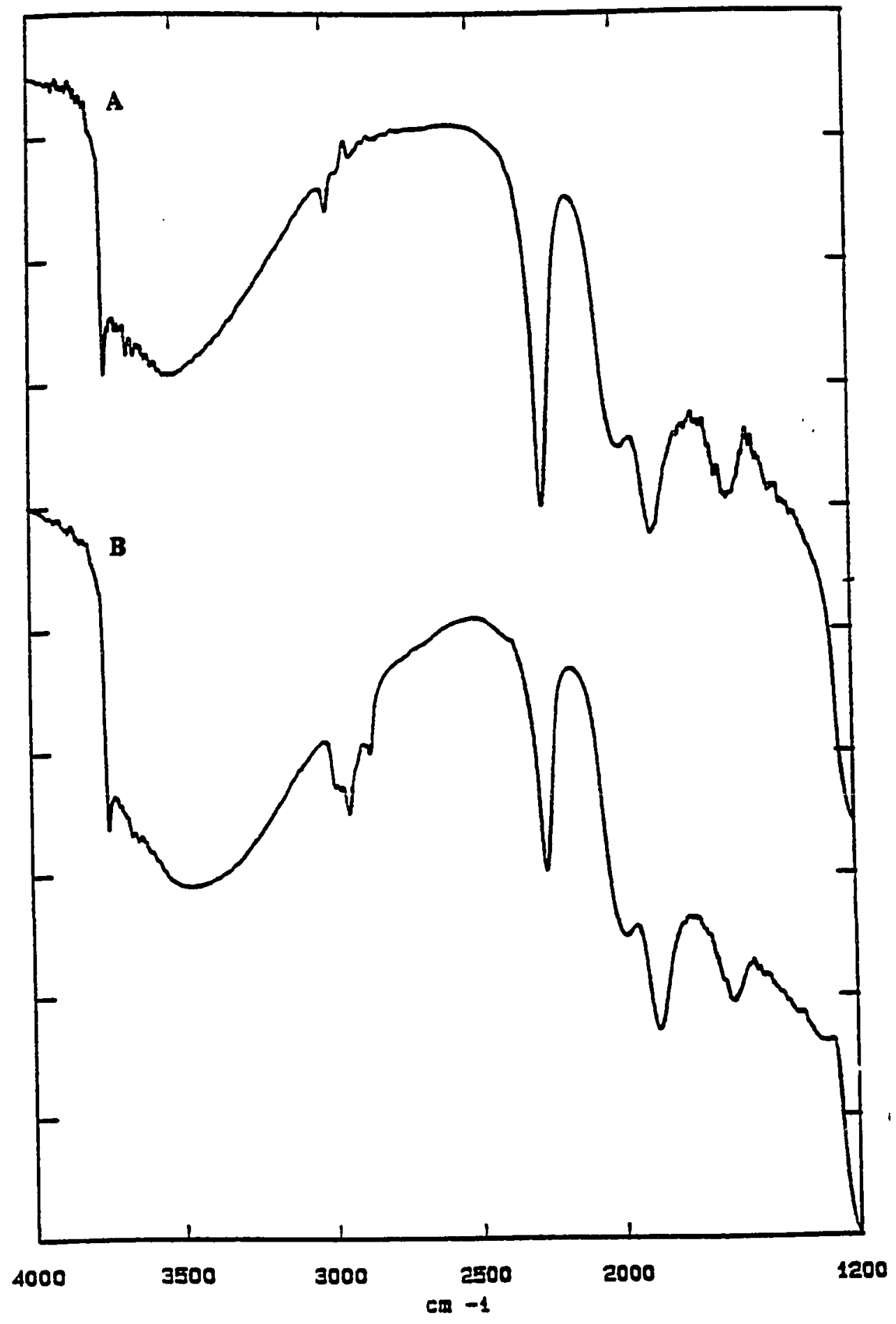


Figure 19. Partial DRIFT spectrum of silane intermediate prepared by silanetriol condensation technique $(A)$, and $(B)$ after bonding with cholesteryl 10-undecenoate.

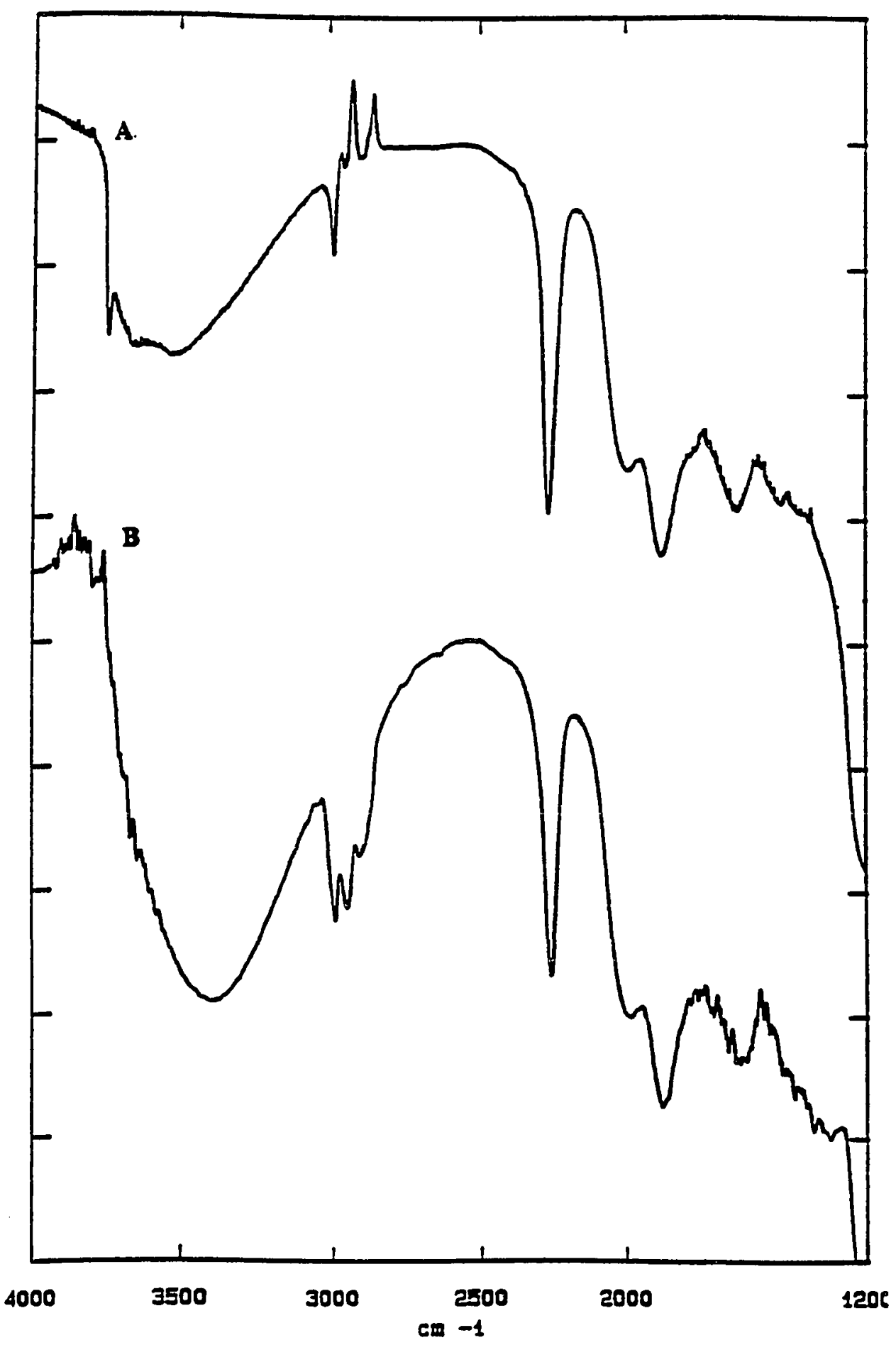


Figure 20. Partial DRIFT spectra of hydrided silica bonded with cholesteryl 10-undecenoate: $(A)$ in hydride intermediate prepared via chlorination/reduction technique, and $(B)$ in hydride intermediate prepared by silanetriol condensation process.

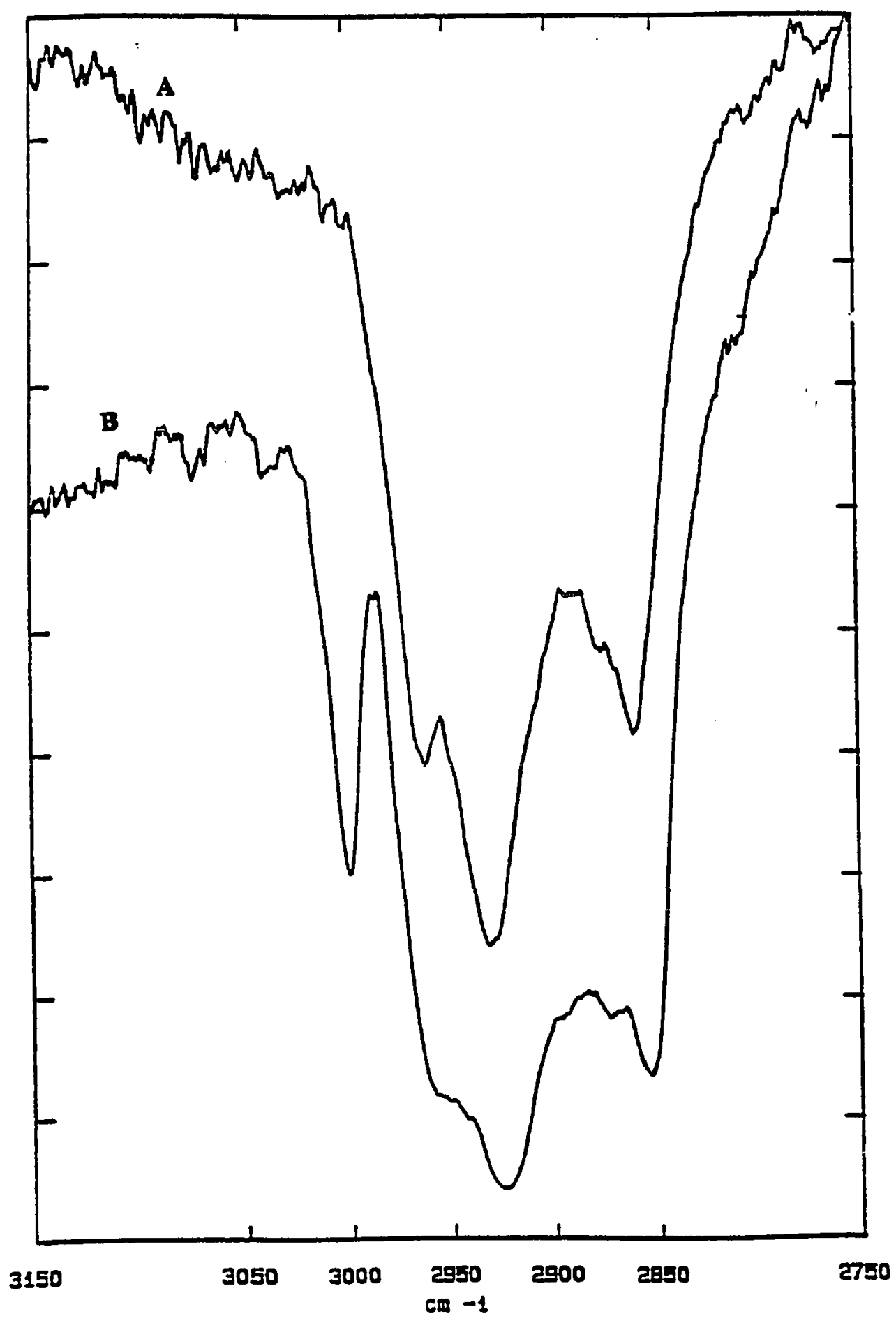




\section{F. Thermal characterization}

Liquid crystalline behavior of both the esters was examined by Differential scanning calorimetry (DSC). It has been shown that 4-methoxyphenyl 4-allyloxy-benzoate (MPAB) developes liquid crystalline behavior when attached to a polysiloxane backbone [26]. The DSC thermogram of MPAB exhibits a single endothermic transition at $90.52{ }^{\circ} \mathrm{C}$ and is shown in Figure 21. Cholesteryl 10undecenoate, on the other hand, exhibits four transitions: first at $90.42{ }^{\circ} \mathrm{C}$ (endothermic), while heating, and three more at $88.41^{\circ} \mathrm{C}, 80.07^{\circ} \mathrm{C}$, and $58.38^{\circ} \mathrm{C}$ (all exothermic) while cooling (Figure 22). The DSC thermogram of MPAB after it was bonded to silica surface exhibits an exothermic transition at $157.15{ }^{\circ} \mathrm{C}$, but no liquid crystalline behavior was observed (Figure 23). The $\alpha_{\mathrm{OH}}$ of silica was 7.6 $\mathrm{mmols} / \mathrm{m}^{2}$ and the surface coverage was $1.4 \mathrm{mmols} / \mathrm{m}^{2}$ (18.5\%) after hydrosilation of MPAB on silica. The DSC thermogram taken here may not be sensitive enough to detect other transitions. Cholesteryl 10-undecenoate shows a surface coverage of $0.3 \mathrm{mmols} / \mathrm{m}^{2}(4 \%)$. The DSC scan of cholesteryl 10-undecenoate did not show any transitions, possibly for the same reason mentioned above. 
Figure 21. DSC thermogram of 4-methoxyphenyl 4-allyloxybenzoate.

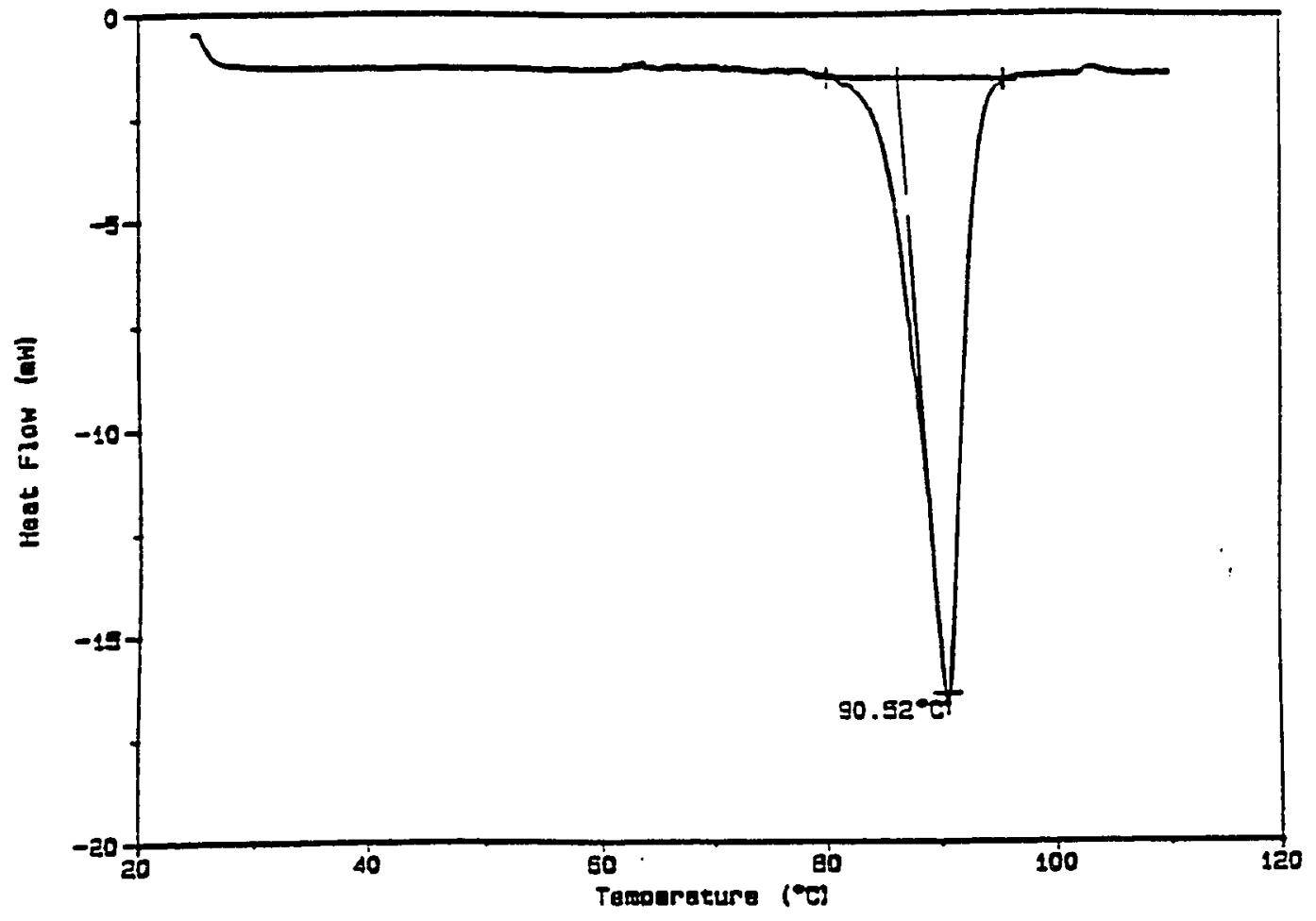


Figure 22 DSC thermogram of cholesteryl 10-undecenoate.

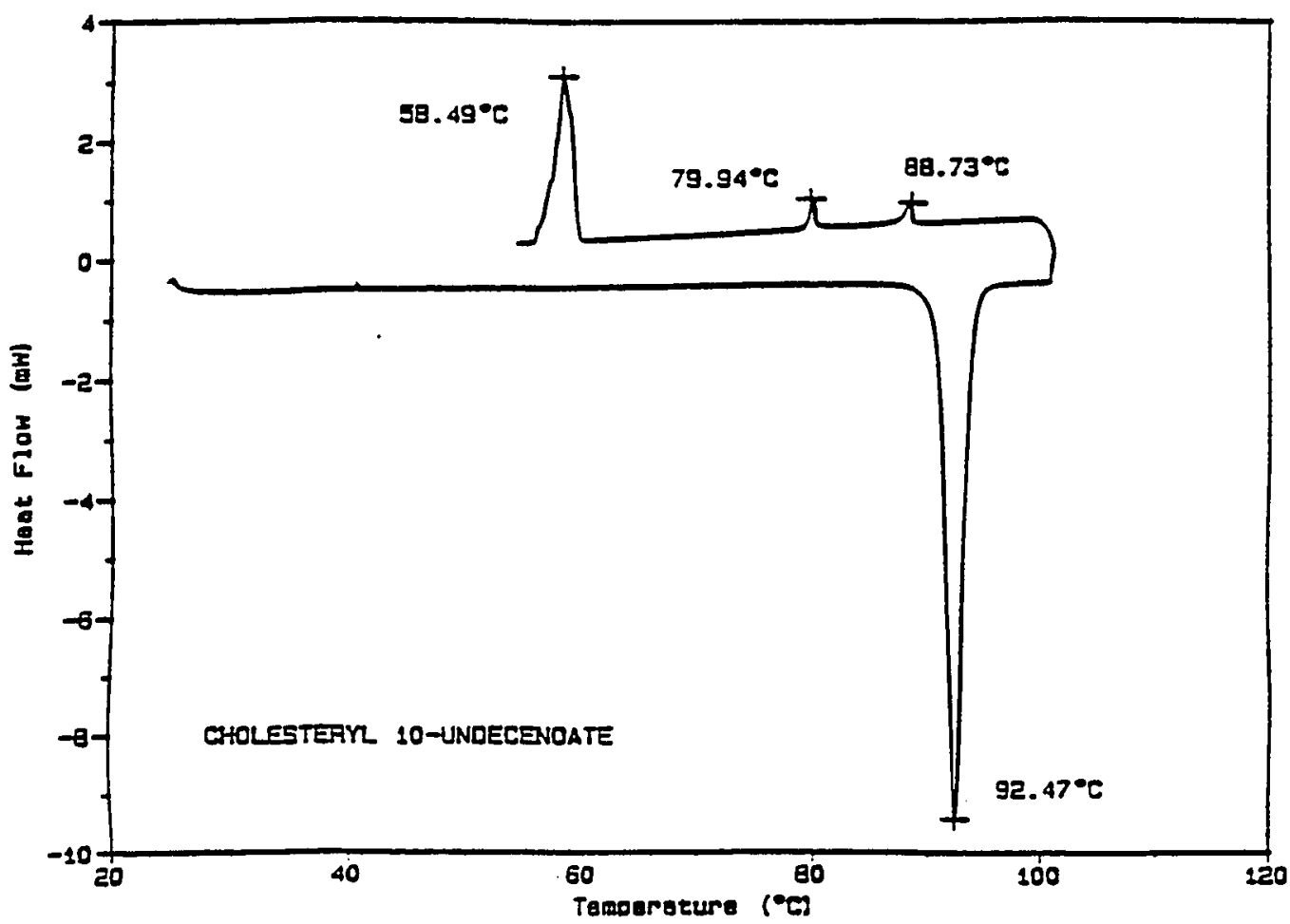


Figure 23. DSC thermogram of 4-methoxyphenyl 4-allyloxybenzoate bonded silica

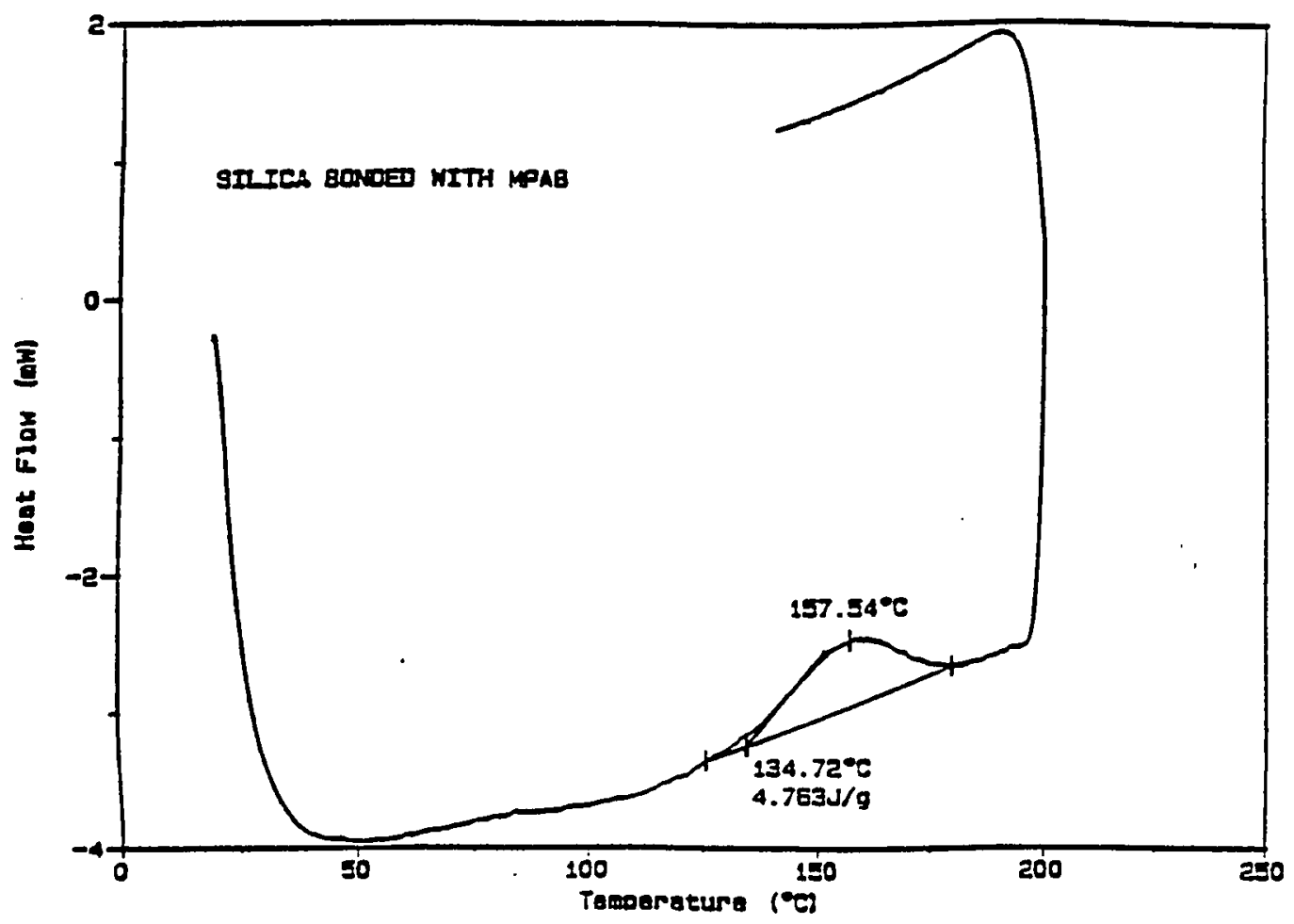




\section{CHAPTER IV}

\section{CONCLUSION}

We have proved that a liquid crystalline compound with a vinyl terminal group can be used to form a covalent bond with the silica surface. In order to achieve a compact geometry of bonded phases, the use of anchored groups like chlorodimethyl silane [29] has been eliminated. The overall yield of Si-C bonding on the silica surface may be improved by the use of alternate catalytic systems and reaction temperature ranges. Comparatively low Si-C bonding could be a physical limit of the silica material used, as well as the size and shape of the liquid crystalline material. Extension of this work should focus on the optimization of the bonding procedure. Bonded liquid crystal phases silica could be used to separate a broad spectrum of optical isomers and/or poly aromatic hydrocarbons. 


\section{REFERENCES}

[1] Halasz and Sebastian, I. Agnew Chem.. (Int. Ed.). 1969, 8, 453.

[2] Deul, H.; Watermann, J.; Hutschneker, K.; Schobinger U.; Gudel, C., Helv.Chim.Acta., 1959, 119, 1160.

[3] Hunter, M. J.; Gordon, M. S.; Barry, A. J.; Hyde, J. F.; Heidenreich, R. D., Ind. Eng. Chem.. 1947, 39, 1389.

[4] Pesek, J. J.; Graham, J. A., Anal. Chem.. 1977, 49, 133.

[5] Pesek, J. J.; Swedberg, S., J. Chromatogr., 1986, 361, 83-91.

[6] Unger, V. K.; Thomas, W.; Adrian, P., Kolloid-Z, 1973, 251, 45-52.

[7] Morrison and Boyd, Organic Chemistry, ELBS, 3rd Ed., 1974

[8] Sandoval, J. E.; Pesek, J. J., Anal. Chem.. 1989, 6I, 2067.

[9] Arkles, B., Petrarch catalog, 1987, S-7, 55.

[10] Pleuddemann, E. P., Silane Coupling Agents, Plenum, N. Y., 1982.

[11] Meier, G., Applications of Liquid Crystals. Springer Veriag, 1975, p. 1.

[12] Janini, G. M., Adv. in Chromalogr., 1979, 17, 233-271. Marcel Dekker.

[13] Witkiweiz, Z. J., Chromatographia. 1982, 251, 311.

[14] Kelkar, H., Ber. Bunsenges. Phys.Chem.. 1963, 67, 698-706.

[15] Kelkar, H.; Fresenius. Z.. Anal. Chem., 1963, 198, 254-266.

[16] Dewar, M. J. S.; Schroeder, P. J., J. Am. Chem. Soc., 1964, 86, 5235-5239.

[17] Dewar, M. J. S; Schroeder J. P., J. Org. Chem., 1965, 30, 3485-3490.

[18] Kelkar, H.; Winterscheidt, W., Anal. Chem., 1960, 1, 220.

[19] Cook, L. E; Sherman, P. L., Anal. Chem., 1974, 46, 122.

[20] Janini, G, M.; Muschik,; G. M.; Zielinski, W. L., Anal. Chem., 1976, 48, 809.

[21] Taylor, P. J.; Sherman, P. L., J. Liquid Chromatogr., 1980, 3, 1.

[22] Taylor, P. J.; Sherman, P. L., J. Liquid Chromatogr., 1986, 3, 21. 
[23] Artaskova, A. A.; Vertova, Z. P.; Yoshin, Y. I., J. Chromatogr., 1986, 365, 27.

[24] Taylor, P. J.; Sherman, P. L., J. Liquid Chromatogr., 1979, 2(9), 1271-1290.

[25] Pesek, J. J.; Siouffi, A. M., Anal. Chem., 1989, 61, 1928.

[26] Apfel, M. A.; Finkelmann, H.: Janini, G. M.; Laub, R. J.; Luthmann, B. H.; Price, A.; Roberts, W. L.; Shaw, T. J.; Smith, C. A., Anal.Chem., 1985. 57, 651-658.

[27] Pesek, J. J.; Cash. T., Chromatogr., 1989, 27, 559-564.

[28] Berendsen and De Galan, J. Liquid Chromatogr, 1978, 1(5), 561-568.

[29] Sandoval, J. E.; Pesek, J. J., Manuscript in preparation. 